\title{
Huanglian Jiedu decoction remodels the periphery microenvironment to inhibit Alzheimer's disease progression based on the "brain-gut" axis through multiple integrated omics
}

Xinru Gu${ }^{1}$, Junyi Zhou', Yanyan Zhou ${ }^{1}$, Hongjie Wang ${ }^{1}$, Nan Si ${ }^{1}$, Wei Ren ${ }^{2}$, Wei Zhao ${ }^{3}$, Xiaorui Fan ${ }^{1}$, Wenya Gao ${ }^{1}$, Xiaolu Wei ${ }^{1}$, Jian Yang ${ }^{1}$, Baolin Bian ${ }^{1 *}$ and Haiyu Zhao ${ }^{1 *}$

\begin{abstract}
Background: In recent years, excellent results have suggested an association between the "brain-gut" axis and Alzheimer's disease (AD) progression, yet the role of the "brain-gut" axis in AD pathogenesis still remains obscure. Herein, we provided a potential link between the central and peripheral neuroinflammatory disorders in AD progression.
\end{abstract}

Methods: The Morris water maze (MWM) test, immunohistochemistry, ELISA, ProcartaPlex Multiplex immunoassay, multiple LC-MS/MS methods, and the V3-V4 regions of 16S rRNA genes were applied to explore potential biomarkers.

Results: In Tg-APP/PS1 mice, gut dysbiosis and lipid metabolism were highly associated with AD-like neuroinflammation. The combination of inflammatory factors (IL-6 and INF- $\gamma$ ), phosphatidylcholines (PCS) and SCFAproducing bacteria were expected to be early diagnostic biomarkers for AD. Huanglian Jiedu decoction (HLJDD) suppressed gut dysbiosis and the associated $A \beta$ accumulation, harnessed neuroinflammation and reversed cognitive impairment.

Conclusion: Together, our findings highlighted the roles of neuroinflammation induced by gut dysbiosis and lipid metabolism disorder in AD progression. This integrated metabolomics approach showed its potential to understand the complex mechanisms of HLJDD in the treatment of AD.

Keywords: Huanglian Jiedu decoction (HLDD), Alzheimer's disease (AD), Gut microflora, Neuroinflammation

\footnotetext{
* Correspondence: blbian@icmm.ac.cn; hyzhao@icmm.ac.cn

'Institute of Chinese Materia Medica, China Academy of Chinese Medical Sciences, Beijing 100700, China

Full list of author information is available at the end of the article
}

C C The Author(s). 2021 Open Access This article is licensed under a Creative Commons Attribution 4.0 International License, which permits use, sharing, adaptation, distribution and reproduction in any medium or format, as long as you give appropriate credit to the original author(s) and the source, provide a link to the Creative Commons licence, and indicate if changes were made. The images or other third party material in this article are included in the article's Creative Commons licence, unless indicated otherwise in a credit line to the material. If material is not included in the article's Creative Commons licence and your intended use is not permitted by statutory regulation or exceeds the permitted use, you will need to obtain permission directly from the copyright holder. To view a copy of this licence, visit http://creativecommons.org/licenses/by/4.0/. The Creative Commons Public Domain Dedication waiver (http://creativecommons.org/publicdomain/zero/1.0/) applies to the data made available in this article, unless otherwise stated in a credit line to the data. 


\section{Introduction}

Alzheimer's disease (AD), a chronic neurodegenerative disease, is a major cause of disability and mortality if not effectively treated. Over 40 million people worldwide are suffering from AD, especially in elderly people [1]. There are currently no preventative or disease-modifying treatments available, despite the countless investments that have been made in the war against AD. Beyond all doubt, the complexity of the aetiology of AD is the biggest challenge to overcome this problem. Now, as many as 1141 anti-AD drugs worldwide were in development. However, only 6 of them stood out and were approved by the Food and Drug Administration (FDA). Regrettably, these drugs are relatively against a single target and are mainly acetylcholinesterase inhibitors, which is not an optimal choice for AD patients with multipathogenesis. GV-971, a mixture of acidic linear oligosaccharides ranging from dimers to decamers (molecular weight up to $\sim 1 \mathrm{kDa}$ ) that were approved by the FDA to carry out a phase 3 clinical for AD in 2020, suppresses gut dysbiosis and the associated phenylalanine/isoleucine accumulation, harnesses neuroinflammation and reverses the cognition impairment [2]. The success of GV971 in anti-AD reveals that multi-target intervention breaks new ground for the drug development of $\mathrm{AD}$. Traditional Chinese medicine (TCM), characterized by multiple components and multiple targets, closely coincides with this strategy.

HLJDD, a classical TCM formula used for fever relief and detoxification, consists of Rhizoma coptidis (Rc), Radix scutellariae (Rs), Cortex phellodendri (Cp) and Fructus Gardeniae (Fg) in a weight ratio of 3:2:2:3. It has been widely applied to treat cerebrovascular diseases, ischaemic stroke, and $\mathrm{AD}$ in many Asian countries for centuries [3-6]. In our previous studies, the chemical profile and haemodynamics of HLJDD were described in detail $[7,8]^{\cdot}$ Sixty-nine compounds in HLJDD were identified, including mainly iridoids, alkaloids and flavonoids. Berberine (Ber), baicalin and geniposide were also representative components [7]. Recently, excellent results on the pharmacological effects of HLJDD or its components have been achieved in the treatment of $\mathrm{AD}$, involving ameliorating cognitive dysfunction, lessening of the plaque burden and oxidative stress and altering lipid metabolism [9, 10]. However, the underlying mechanisms of HLJDD on the amelioration of AD are still a mystery.

Neuroinflammation is a key factor in the neurodegenerative process of AD [11]. This process involves an initial inflammatory stimulus $(\mathrm{A} \beta$, pro-inflammatory cytokines, chemokines and the generation of reactive oxygen species) that triggers microglia, the resident macrophage within the central nervous system (CNS) [12-15]. In addition, monocytes recruited from the periphery can interact with microglia to influence the expression of amyloid-beta $[16,17]$. So the accumulation of $A \beta$ throughout the brain is the failure of microglia and peripherical monocytes to remove extracellular amyloid [18, 19], which reversely activates microglia and releases inflammatory cytokines, such as interleukin-1 $\beta$ (IL-1 $\beta$ ), IL-6 and tumour necrosis factor (TNF- $\alpha$ ) [20]. In addition, they secrete oxidative stress-related molecules, such as nitric oxide (NO), reactive oxygen species (ROS) and superoxide anions [21]. Glucose is normally the major energy source for the brain, but in $\mathrm{AD}$, glucose metabolism dramatically decreases [15, 22-24]. However, docosahexaenoic acid (DHA) facilitates the transport of glucose into the brain by regulation of glucose transporter protein-1 (GLUT1) transporters and reducing $A \beta$ plaque aggregation in individuals with moderate dementia and AD [25]. Furthermore, phospholipids (PLs), which act as storage depots from a complex meshwork of lipid mediators in cell membranes, are especially important in controlling neuroinflammation [26]. PLs influence the formation of $A \beta$ peptides by affecting membrane proteins, such as APP, and $\beta$ - and $\gamma$ secretase. The decrease of PCs in both the plasma and the brain are associated with impaired cognitive performance in elderly people and AD patients [27, 28].

It should be noted that neuroinflammation is not solely restricted to contributions from resident biochemical factors in the brain, as perturbations in microbial diversity are related to propagating neuroinflammation in preclinical models of AD. Gut microbiota could affect the immune system directly via activation of the vagus nerve $[29,30]$, which in turn triggers bidirectional communication with the CNS and links them to the cognitive and emotional centres of the brain [31-33]. The results of a recent clinical trial performed on elderly subjects with dementia support the evidence of the role of amyloid and related bacterial accumulation in the pathogenesis of cognitive damage [34]. Additionally, the dysregulation of gut microbiota is responsible for the increased permeability of intestinal barriers and the blood-brain barrier (BBB) [35]. Short-chain fatty acids (SCFAs) produced by bacterial fermentation of dietary carbohydrates can cross the $\mathrm{BBB}$, modulate brain development and behaviour and regulate microglia homoeostasis [36-38]. In addition to SCFAs, gut bacteria also produce a range of substances with neuroactivity and immunomodulatory effects, including dopamine, $\gamma$-aminobutyric acid (GABA) and histamine [39-41]. Tryptophan (Trp) is metabolized in the periphery within the gut neurons and enterochromaffin cells, and centrally in the neurons of the raphe of the brain stem [42]. Dysregulation of Trp metabolism causes shifts in the balance between the Kyn and 5-HT pathways and is associated with psychiatric and neurological disorders [43]. 
Bile acids (BAs) are critically important for the maintenance of a healthy gut microbiota, a balance between lipid and carbohydrate metabolism, energy homeostasis and innate immunity [44, 45]. Disturbance of BAs has been demonstrated in AD progression [46, 47]. Circulating BAs were thought to provide a communication bridge between the gut and the brain, and their alteration reflects gut dysbiosis [48-50]. Bile salt hydrolase-rich (BSH) bacteria readily modify the BA profile. In turn, intestinal BAs control the growth and maintenance of commensal bacteria [51-53]. Metagenomic analyses have demonstrated that functional BSH is present in all major bacterial divisions and archaeal species in the human gut, including members of Lactobacilli, Bifidobacteria, Clostridium and Bacteroides [51, 54-56].
Plenty of evidence shown that peripheral and central inflammations play an important role in the pathogenesis of AD. The hypothesis of "brain-gut" axis will be an useful and promising exploration. In this study, we aimed to reveal the phenotypic features of APP/PS1 mice as comprehensively as possible, and provide a potential effective, safe and economical intervention strategy from TCM.

\section{Results}

\section{Cognitive deficiency in Tg-APP/PS1 mice}

The cognitive functions of 8-month-old Tg-APP/PS1 (Tg) mice were significantly impaired compared to agematched wild-type (WT) mice (Fig. 1). In the navigation test, $\mathrm{Tg}$ mice normally took longer time to reach the platform as compared to the WT mice over a 3-day

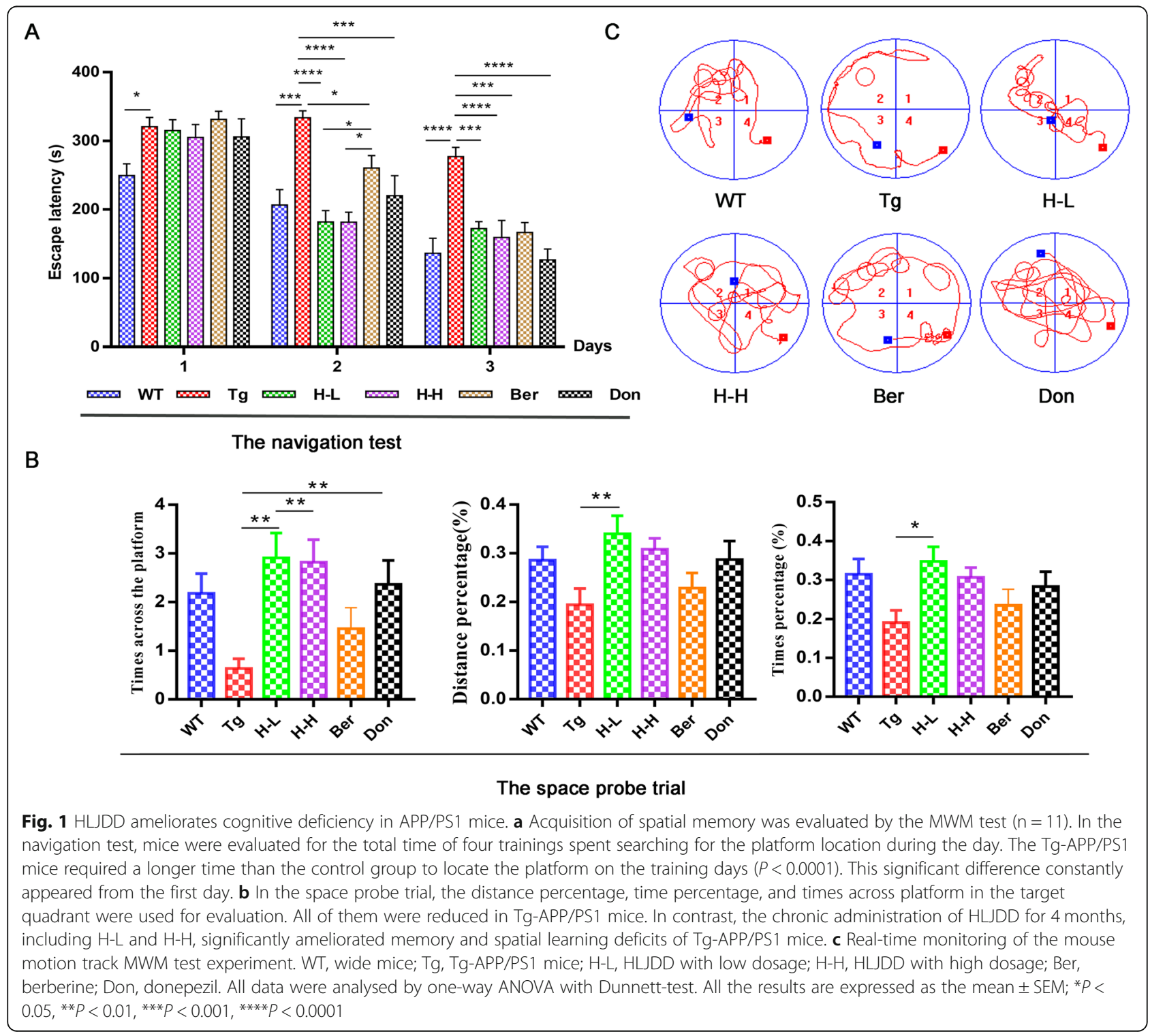


training period $(P<0.0001)$ (Fig. 1a). In the space probe test, the times across the platform was reduced to more than half of that in WT mice (Fig. 1b). Meanwhile, the trajectory map of $\mathrm{Tg}$ mice was disorganized and purposeless (Fig. 1c). A $\beta$ plaques markedly accumulated in the brain cortex and hippocampus of Tg mice compared with WT mice. Congo red staining has shown that $A \beta$ deposition with higher density and larger area existed in the hippocampus and cortex of $\mathrm{Tg}$ mice than that of WT mice (Fig. 2a). Meanwhile, the results of immunohistochemistry in the hippocampus and cortex of $\mathrm{Tg}$ mice were also positive (Fig. 2b, c). Furthermore, the brain contents of SOD dramatically decreased in $\mathrm{Tg}$ mice $(P<0.001)$ (Fig. $2 \mathrm{~d})$. Chronic oral administration of $\mathrm{H}-\mathrm{L}$ (HLJDD with low dosage: $172 \mathrm{mg} / \mathrm{kg} /$ day) and $\mathrm{H}-\mathrm{H}$ (HLJDD with high dosage: $344 \mathrm{mg} / \mathrm{kg} /$ day) for 4 months significantly ameliorated the memory and spatial learning deficits of $\mathrm{Tg}$ mice by suppressing the accumulation of $\mathrm{A} \beta$ plaques in the cortex and hippocampus. In addition, H-L could increase the level of SOD in the brain of $\mathrm{Tg}$ mice to 1.23 -fold and has a tendency to downregulate MDA. Therefore, we speculated that HLJDD has the potential to attenuate oxidative stress.

\section{The CNS neuroinflammation in Tg-APP/PS1 mice Changes in inflammatory cytokines}

Cytokines of total brain homogenates were measured by ProcartaPlex Multiplex Immunoassay to further assess the neuroinflammatory profile (Fig. 3a). Compared with WT mice, IFN- $\gamma$ and IL-12p70 were significantly decreased in $\mathrm{Tg}$ mice with the content of 7.75 and 38.98 $\mathrm{pg} / \mathrm{g}$. The content of IL-6 in Tg mice was about 1.4 times greater than that in WT mice. After the administration of HLJDD, the levels of IFN- $\gamma$ tended to be a normal value (H-L: $9.02 \mathrm{pg} / \mathrm{g} ; \mathrm{H}-\mathrm{H}: 8.22 \mathrm{pg} / \mathrm{g}$ ), and the level of IL-6 observably decreased $(P<0.05)$. Furthermore, anti-inflammatory cytokine IL-4 and IL-10 both considerably increased in HLJDD mice compared with Tg mice.

\section{Disorder of endogenous metabolites}

Increasing evidence suggests that metabolic perturbations in various pathways mediate the occurrence of $\mathrm{AD}$ pathology as well as the onset of cognitive impairment in patients. UPLC-QQQ MS/MS was employed to evaluate the alteration of endogenous metabolites in Tg mice. Overall, there was no significant fluctuation in the neurotransmitters (NTs) levels in the brain of $\mathrm{Tg}$ mice compared with WT mice (Table 1). However, the levels of citrulline and methionine in $\mathrm{Tg}$ mice were higher than that in WT mice (fold: 1.66 and 1.28, respectively). HLJDD significantly increased the levels of NTs in the brain of Tg mice, including essential amino acids (phenylalanine: Phe, Trp, leucine, isoleucine and threonine), proline, choline, glutamate (Glu) and GABA, and arginine, tyrosine and asparaginate. Simultaneously, L-cysteine decreased after intervention with HLJDD. As far as PUFAs (polyunsaturated fats) were concerned (Fig. 3b), the overall pattern of results showed no significant differences in brain PUFAs of Tg mice relative to WT mice. Nevertheless, treatment with HLJDD significantly increased the brain levels of arachidonic acid (AA), DHA, eicosapentaenoic acid (EPA), linoleic acid (LA) and oleic acid (OLA) and decreased the content of $\gamma$-linolenic acid (GLA). Moreover, HLJDD treatment inhibited cyclooxygenase $2(\mathrm{COX}-2)$ and 5lipoxygenase (5-LOX) expression in the brain of $\mathrm{Tg}$ mice (Fig. 3c).

In addition, dysregulation of lipid metabolism in the brains of Tg mice was confirmed by supervised OPLSDA with the values of $R^{2} Y$ and $Q^{2}(93 \%$ and $79 \%$, respectively). In the PLS-DA plots (Fig. 3d), the HLJDD administration groups were relatively independent and had no intersection with $\mathrm{Tg}$ mice, but were closer to WT mice. In detail, 40 pathological lipid biomarkers in brain tissue were identified (Table 2). The levels of $17 /$ 21 PCs, 5/5 PEs, 3/3 glucosylceramides (GlcCers), 4/4 ceramides (Cers) and 5/7 sphingomyelins (SMs) were lower in Tg mice than in WT mice. HLJDD attenuated this lesion by increasing the contents of PCs and PEs in the brains of mice.

\section{The peripheral inflammation in Tg-APP/PS1 mice Changes in cytokines}

As shown in Fig. 4a, the levels of IFN- $\gamma$, IL-13 and IL$12 \mathrm{P} 70$ in the serum of $\mathrm{Tg}$ mice $(0.96,0.65$ and $0.34 \mathrm{pg} /$ $\mathrm{mL}$, respectively) were lower than these in WT mice (2.58, 1.08 and $0.47 \mathrm{pg} / \mathrm{mL}$, respectively), while the contents of monocyte chemotactic protein 1 (MCP-1, 2.53fold) and IL-6 (1.43-fold) were higher. Meanwhile, we found that the change tendency of IFN- $\gamma$, IL- 6 and IL12p70 in the periphery of $\mathrm{Tg}$ mice were consistent with those in the brain. Oral administration of HLJDD suppressed pro-inflammatory cytokines IL-1 $\beta$, IL-6, MCP-1 and TNF- $\alpha$ expression in the serum of Tg mice.

\section{Alteration of endogenous metabolites}

An overall level of peripheral omega- 6 acid and omega-9 acid decreased in $\mathrm{Tg}$ mice compared to WT mice (Fig. 4b), including LA $(P<0.05)$, AA $(P<0.001)$, EPA $(P<0.05)$, DHA (reduced by $25 \%)$, GLA (reduced by $28 \%)$ and OLA $(P<0.05)$. Nevertheless, the levels of serum of PUFAs showed no significant changes after HLJDD. PUFAs are required for maintaining the structure, function and vascular integrity of the brain. Nonessential fatty acids are synthesized in the brain, but essential PUFAs (e.g., AA, DHA and EPA) are largely acquired from the peripheral circulation [3]. Therefore, to investigate whether HLJDD affected central PUFAs in Tg mice 


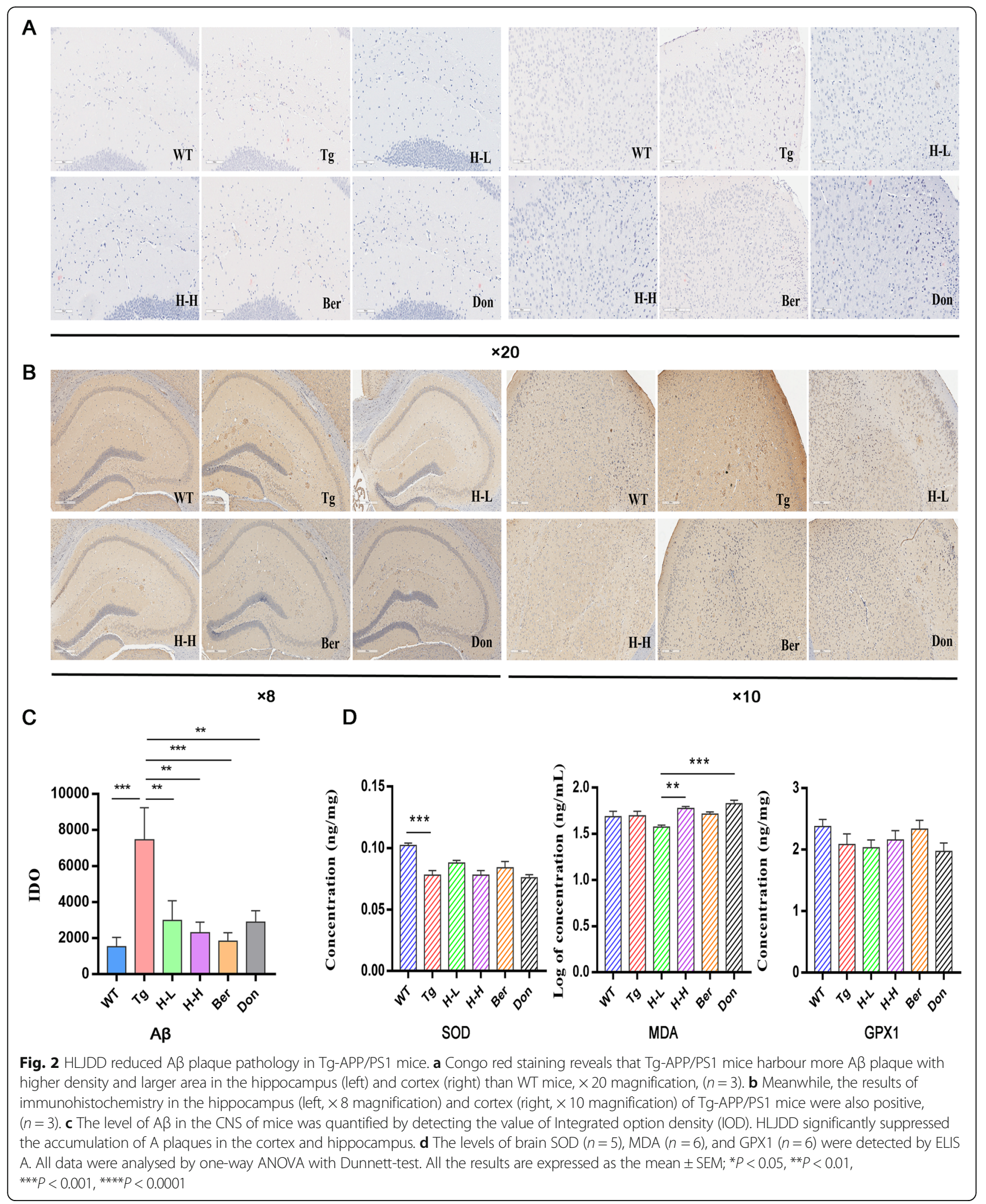

by regulating peripheral PUFAs, correlation network analysis was employed based on the measured contents (Fig. 4c). Results showed that the contents of serum
PUFAs (DHA, EPA, LA and GLA) were negatively correlating with those of central PUFAs, and the level of serum AA was a positive correlation with that of central AA. 


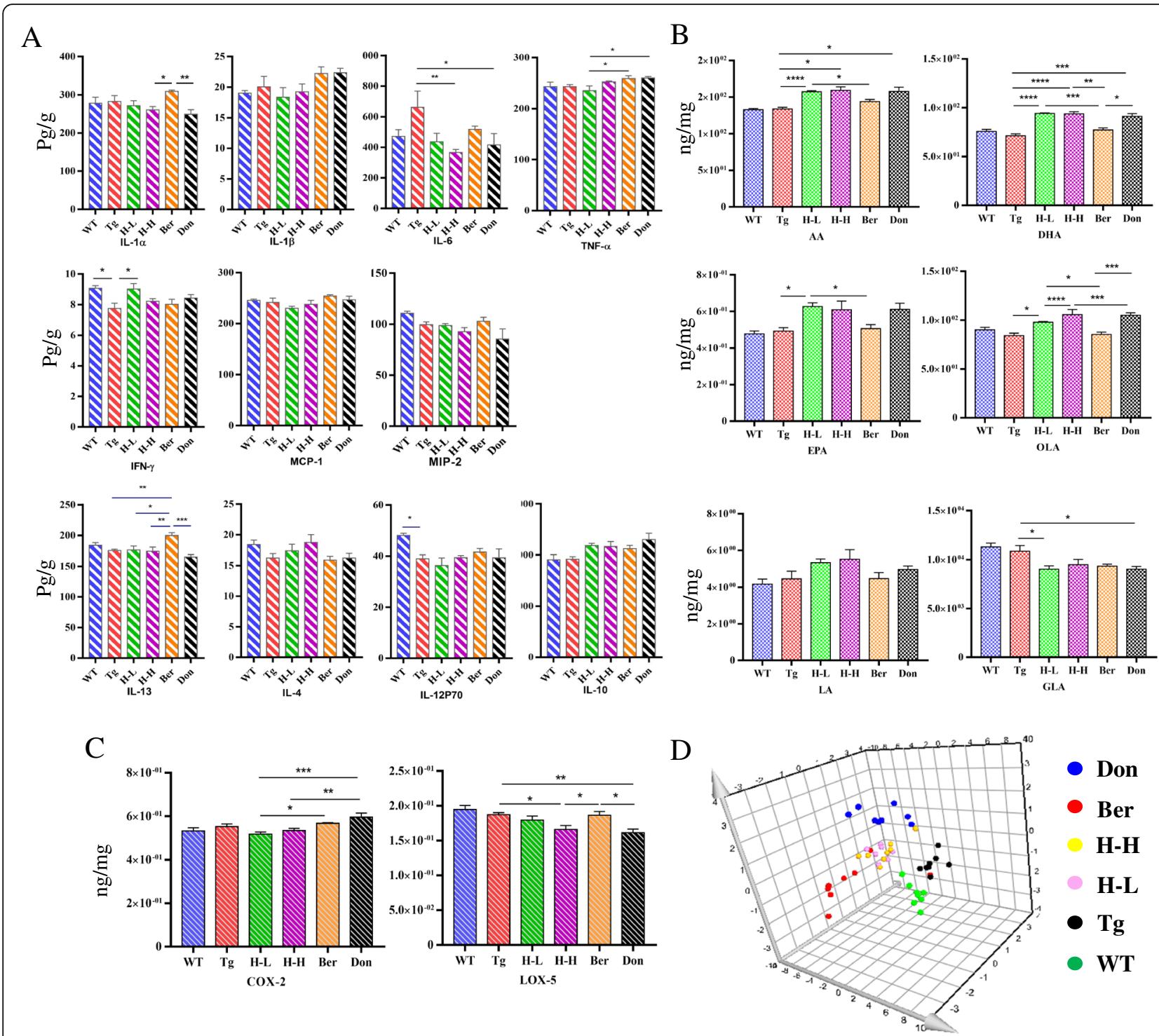

Fig. 3 HLDD improved the CNS inflammatory microenvironment in Tg-APP/PS1 mice. a Changes in pro-inflammatory and anti-inflammatory factors $(n=5)$. b Changes in UFAs $(n=6)$. c Changes in COX-2 and 5-Lox $(n=5)$. d PLS-DA plot. All data were analysed by one-way ANOVA with Dunnett-test. All the results are expressed as the mean $\pm \mathrm{SEM}$; ${ }^{*} P<0.05$, ${ }^{* *} P<0.01$. ${ }^{* *} P<0.001$, ${ }^{* * *} P<0.0001$

Dysregulation of lipid metabolism in the serum of Tg mice was also confirmed by OPLS-DA, with values of $R^{2} x$ and $Q^{2}$ of $72 \%$ and $40 \%$, respectively. The PLS-DA plot showed that HLJDD groups (including $\mathrm{H}-\mathrm{L}$ and $\mathrm{H}-\mathrm{H}$ ) were relatively independent and had no intersection with the WT and Tg groups (Fig. 4d), which was similar to the pattern in the brain. In detail, 13 LP biomarkers in serum were screened, including $11 \mathrm{PCs}, 1 \mathrm{PE}$ and 1 GlcCers (Table 3). Almost all serum lipid biomarkers decreased in Tg mice compared with WT mice. Interestingly, all of these biomarkers were reversed by HLJDD.

Additionally, we found that some significant changes occurred in peripheral BAs in Tg mice compared with
WT mice (Table S1). CA was hardly detected in the periphery of WT mice, but they remained high in the serum of AD mice. An increase of tauro-conjugated BAs (taurodeoxycholic acid (TDCA), tauroursodeoxycholic acid (TUDCA), taurohyodeoxycholic acid (THDCA), tauro- $\alpha$ muricholic acid (T- $\alpha-\mathrm{MCA})$ ) and deoxycholic acid (DCA, almost a 30-fold increase) were observed in $\mathrm{Tg}$ mice compared with WT. Interestingly, HLJDD significantly decreased the levels of DCA, T-MCA and THDC A. It has been reported that the ratio between BAs reflected enzymatic activities in the liver and the gut microbiome. The cholic acid (CA) to chenodesoxycholic acid (CDCA) ratio reflected if a possible shift in BA 
Table 1 The effect of HLJDD on neurotransmitters in the CNS of Tg-APP/PS1 mice

\begin{tabular}{|c|c|c|c|c|c|c|}
\hline $\begin{array}{l}\text { Chemical } \\
\text { compounds }\end{array}$ & WT & $\mathrm{Tg}$ & H-L & H-H & Ber & Don \\
\hline Phe & $56.07 \pm 4.23$ & $62.89 \pm 2.63$ & $91.19 \pm 8.08 \#$ & $100.73 \pm 12.32$ & $62.66 \pm 1.47$ & $87.95 \pm 2.55 \#$ \\
\hline Proline & $96.44 \pm 5.78$ & $106.26 \pm 3.59$ & $151.27 \pm 14.16 \#$ & $170.69 \pm 22.53 \# \#$ & $104.89 \pm 1.88$ & $147.49 \pm 3.48$ \\
\hline Citrulline & $17.14 \pm 1.26$ & $28.52+3.12 * *$ & $25.19 \pm 1.95$ & $27.17 \pm 3.58$ & $15.48 \pm 0.37$ \# & $25.41 \pm 1.77$ \\
\hline Glu & $574.73 \pm 28.65$ & $586.17 \pm 28.80$ & $685.28 \pm 50.03$ & $804.81 \pm 100.45 \#$ & $576.31 \pm 17.52$ & $749.79 \pm 35.7$ \\
\hline Glutamine & $1202.48 \pm 137.89$ & $1347.38 \pm 21.86$ & $1163.28 \pm 91.38$ & $1480.31 \pm 186.45$ & $1260.20 \pm 24$ & $1273.86 \pm 60.45$ \\
\hline Arginine & $165.41 \pm 6.95$ & $186.76 \pm 5.56$ & $249.49 \pm 24.78 \#$ & $298.36 \pm 37.21 \ldots \#$ & $181.35 \pm 13.69$ & $249.48 \pm 8.13 \#$ \\
\hline Tyrosine & $79.26 \pm 4.18$ & $83.14 \pm 3.19$ & $121.53 \pm 9.6$ \# & $134.78 \pm 17.69 \ldots \#$ & $82.69 \pm 1.01$ & $123.56 \pm 6.11$ \# \\
\hline Taurine & $1607.52 \pm 48.78$ & $1771.33 \pm 23.5$ & $1919.82 \pm 123.45$ & $2063.9 \pm 266.57$ & $1804.62 \pm 26.06$ & $2067.54 \pm 60$ \\
\hline Trp & $19.79 \pm 1.28$ & $22.49 \pm 1.09$ & $33.03 \pm 2.54 \#$ & $34.92 \pm 4.34$ \#\# & $22.98 \pm 0.67$ & $31.25 \pm 1.33 \#$ \\
\hline Serine & $195.3 \pm 10.8$ & $219.52 \pm 6.18$ & $284.3 \pm 22.44$ & $306.18 \pm 38.51 \#$ & $199.22 \pm 5.35$ & $287.88 \pm 11.89$ \# \\
\hline GABA & $825.51 \pm 24.68$ & $911.25 \pm 27.32$ & $1031.57 \pm 77.17$ & $1176.78 \pm 134.08$ \# & $907.7 \pm 13.14$ & $1120.63 \pm 35.53$ \\
\hline Asparaginase & $2.60 \mathrm{E}-2 \pm 1.01 \mathrm{E}-3$ & $3.08 \mathrm{E}-2 \pm 1.17 \mathrm{E}-3$ & $\begin{array}{c}4.21 \mathrm{E}-2 \pm 2.01 \mathrm{E}-3 \\
\# \# \#\end{array}$ & $\begin{array}{c}4.57 \mathrm{E}-2 \pm 1.43 \mathrm{E}-3 \\
\# \# \#\end{array}$ & $3.08 \mathrm{E}-2 \pm 7.73 \mathrm{E}-4$ & $\begin{array}{c}4.34 \mathrm{E}-2 \pm 2.32 \mathrm{E}-3 \\
\# \# \#\end{array}$ \\
\hline Choline & $23.65 \pm 0.75$ & $25.42 \pm 0.4$ & $26.7 \pm 0.64$ & $31.95 \pm 3.03$ \#\# & $25.63 \pm 0.33$ & $29.87 \pm 0.93$ \\
\hline L-cysteine & $5.77 \mathrm{E}-2 \pm 3.25 \mathrm{E}-3$ & $5.11 \mathrm{E}-2 \pm 4.72 \mathrm{E}-3$ & $\begin{array}{c}1.7 \mathrm{E}-2 \pm 1.72 \mathrm{E}-3 \\
\ldots \# \#\end{array}$ & $\begin{array}{c}7.84 \mathrm{E}-3 \pm 7.68 \mathrm{E}-4 \\
\ldots \# \#\end{array}$ & $\begin{array}{c}2.79 \mathrm{E}-2 \pm 1.29 \mathrm{E}-3 \\
\# \# \#\end{array}$ & $\begin{array}{c}5.3 \mathrm{E}-3 \pm 4.91 \mathrm{E}-4 \\
\# \# \#\end{array}$ \\
\hline L-isoleucine & $1.62 \pm 0.1$ & $1.91 \pm 0.07$ & $2.81 \pm 0.22 \# \#$ & $3.24 \pm 0.28$ \#\#\# & $1.86 \pm 0.04$ & $2.74 \pm 0.13 \#$ \\
\hline L-leucine & $3.46 \pm 0.18$ & $4.15 \pm 0.12$ & $5.88 \pm 0.1 \#$ & $5.61 \pm 0.55 \#$ & $4.04 \pm 0.06$ & $5.67 \pm 0.24 \ldots \#$ \\
\hline Methionine & $0.56 \pm 0.017$ & $0.72 \pm 0.023$ & $0.69 \pm 0.021$ & $0.66 \pm 0.114$ & $0.55 \pm 0.011$ & $0.76 \pm 0.039$ \\
\hline Threonine & $0.26 \pm 0.013$ & $0.28 \pm 0.008$ & $0.38 \pm 0.009 \#$ & $0.46 \pm 0.04 \ldots \# \#$ & $0.26 \pm 0.004$ & $0.41 \pm 0.017$ \#\# \\
\hline Urea & $13.95 \pm 0.24$ & $14.45 \pm 0.14$ & $13.22 \pm 0.5$ & $14.83 \pm 0.21$ & $12.91 \pm 0.39 \#$ & $15.28 \pm 0.52$ \\
\hline
\end{tabular}

Changes in NTs $(n=6-8)$, absolute quantification $(\mu \mathrm{g} / \mathrm{g})$; the remaining NTs were measured in relative quantities $\left(A_{s} / A_{s}\right)$; colour coded according to the contents. The colour from light to dark represents the content from low to high. All data were analysed by one-way ANOVA with Dunnett-test. All the results are expressed

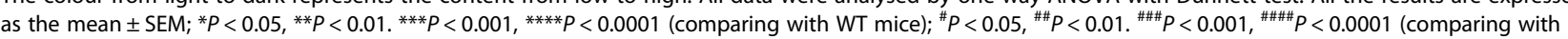
Tg mice)

synthesis from the primary to the alternative BA pathway has occurred in the liver. Ratios of secondary to primary BAs was used to assess the activity of intestinal microbiome enzymes which influenced the production of secondary BAs [47]. In this study, the ratio of CA: CDCA and the secondary to primary BAs were higher in $\mathrm{Tg}$ mice than those in WT mice, which were significantly reversed by HLJDD.

\section{Alteration of the gut microbiota in Tg-APP/PS1 mice}

Pyrosequencing was employed to monitor the faecal microbiota composition in Tg mice. Bacterial richness and $\alpha$-diversity in $\mathrm{Tg}$ mice showed no significant differences relative to WT, as demonstrated by the rarefaction curve, Chao index, and Shannon index (Fig. S1a-c). Moreover, HLJDD treatment did not affect the diversity of microflora in Tg mice, but Ber treatment remarkably decreased the levels of the Shannon index. According to ANOSIM analysis, the intra-group difference was less than the inter-group difference. (Fig. S1d). For PLS-DA analysis (Fig. S1e), the faecal microbiota composition of $\mathrm{Tg}$ mice was significantly different from that of WT mice. However, HLJDD could shift the gut microbiota composition. At the phylum level (Fig. S1f), Tg mice exhibited Firmicutes populations more abundantly than WT (41\% and 35\%, respectively), as previously reported $[57,58]$, while the Bacteroidetes population was lower (54\%, 60\% respectively) [59]. Treatment with HLJDD in Tg mice decreased the population of Firmicutes and increased the population of Proteobacteria. At the family level (Table 4), higher relative abundances of Lachnospiraceae (1.24-fold), Rikenellacae (1.3-fold) and Porphyromonadaceae (2.24-fold) were observed in $\mathrm{Tg}$ mice than those in WT, while the relative abundances of Bacteroidales_S24-7_group, Coriobacteriaceae and Alcaligenaceae were lower (Tg: 4.13\%, 0.13\%, 0.16\%; WT: $5.37 \%$, $0.29 \%, 0.58 \%)$. Treatment with HLJDD increased the relative abundances of Prevotellaceae, Lactobacillaceae, Peptococcaceae, Alcaligenaceae and Helicobacteraceae and reduced the relative abundances of Bacteroidales S24-7_group, Lachnospiraceae and Porphyromonadaceae. At the genus level (Table 5), the relative abundances of unidentified, Parasutterella, Blautia, LachnospiraceaeUCG-001 and Ruminococcaceae-UCG-014 were lower in 
Table 2 The metabolic changes of 40 potential lipids markers in the CNS of Tg mice

\begin{tabular}{|c|c|c|c|c|c|c|c|c|c|c|c|c|c|}
\hline \multirow{2}{*}{$\begin{array}{l}\text { Group } \\
\text { PC(18:0/18:2) }\end{array}$} & \multirow{2}{*}{$\begin{array}{l}\text { Actua M (m/z) } \\
786.6007\end{array}$} & \multirow{2}{*}{$\begin{array}{l}t_{R}(\text { mine }) \\
12.13\end{array}$} & \multirow{2}{*}{$\begin{array}{l}\text { T-test } \\
\text { P-value }\end{array}$} & $\begin{array}{l}\text { WT } \\
/ \text { Tg }\end{array}$ & \multicolumn{3}{|c|}{$\begin{array}{l}\mathrm{H}-\mathrm{L} \\
/ \mathrm{Tg}\end{array}$} & \multicolumn{2}{|l|}{$\begin{array}{l}\mathrm{H}-\mathrm{H} \\
/ \mathrm{Tg}\end{array}$} & $\begin{array}{l}\text { Ber } \\
/ \mathrm{Tg}\end{array}$ & \multicolumn{3}{|c|}{$\begin{array}{l}\text { Don } \\
\text { / } \mathrm{Tg}\end{array}$} \\
\hline & & & & 1.15 & $*$ & 1.18 & $\# \#$ & 1.20 & \#\# & 1.36 & \#\#\# & 1.15 & $\#$ \\
\hline $\operatorname{PC}(22: 6 / 18: 0)$ & 834.6007 & 11.75 & $1.85 \mathrm{E}-05$ & 1.19 & $* * *$ & 1.15 & \#\# & 1.18 & \#\#\# & 1.29 & \#\#\#\# & 1.25 & \#\#\#\# \\
\hline PC(20:0/18:1) & 816.6477 & 14.91 & $1.24 \mathrm{E}-04$ & 1.16 & $* * *$ & 1.05 & l & 1.03 & I & 1.21 & \#\#\# & 1.09 & / \\
\hline $\operatorname{PC}(20: 4 / 20: 3)$ & 832.5851 & 10.59 & $1.25 \mathrm{E}-04$ & 1.14 & ** & 1.05 & / & 1.09 & I & 1.10 & $\#$ & 1.09 & I \\
\hline PC(18:0/18:0) & 790.6320 & 14.81 & 4.69E-04 & 1.11 & ** & 0.75 & \#\#\# & 0.75 & \#\#\#\# & 0.81 & \#\#\# & 0.87 & \#\#\# \\
\hline $\operatorname{PC}(18: 0 / 22: 5)$ & 836.6164 & 12.21 & $6.16 \mathrm{E}-04$ & 1.11 & / & 1.11 & I & 1.14 & \#\# & 1.24 & \#\#\# & 1.11 & I \\
\hline $\operatorname{PC}(18: 0 / 20: 2)$ & 814.6320 & 13.65 & $2.33 \mathrm{E}-03$ & 1.10 & I & 1.11 & $\# \#$ & 1.08 & I & 1.24 & \#\#\# & 1.08 & I \\
\hline $\operatorname{PC}(18: 1 / 17: 0)$ & 774.6007 & 12.77 & $1.89 \mathrm{E}-02$ & 1.10 & I & 1.09 & ' & 1.12 & I & 1.19 & \#\# & 1.05 & I \\
\hline $\operatorname{PC}(22: 6 / 17: 0)$ & 820.5851 & 11.07 & $2.59 \mathrm{E}-03$ & 1.15 & / & 1.08 & l & 1.14 & I & 1.11 & l & 1.28 & $\# \#$ \\
\hline $\operatorname{PC}(16: 0 / 18: 1)$ & 760.5851 & 11.94 & $7.51 \mathrm{E}-05$ & 1.19 & $* *$ & 1.18 & $\#$ & 1.23 & \#\#\# & 1.42 & \#\#\# & 1.21 & \#\# \\
\hline $\operatorname{PC}(16: 0 / 16: 0)$ & 734.5694 & 11.74 & $1.63 \mathrm{E}-04$ & 1.21 & $* *$ & 1.08 & I & 1.12 & I & 1.25 & \#\# & 1.14 & 1 \\
\hline PC(18:0/18:1) & 788.6164 & 13.56 & 4.16E-04 & 1.08 & ' & 1.10 & \# & 1.10 & I & 1.26 & \#\#\# & 1.09 & / \\
\hline $\operatorname{PC}(20: 4 / 18: 2)$ & 806.5694 & 10.46 & $7.91 \mathrm{E}-04$ & 1.11 & * & 1.01 & I & 1.05 & 1 & 1.08 & I & 1.06 & 1 \\
\hline PC(16:0/20:4) & 782.5694 & 10.77 & $1.15 \mathrm{E}-02$ & 1.10 & 1 & 1.02 & 1 & 1.07 & I & 1.14 & \# & 1.10 & 1 \\
\hline $\operatorname{PC}(18: 0 / 16: 0)$ & 762.6007 & 13.32 & $1.28 \mathrm{E}-02$ & 1.09 & 1 & 0.88 & \# & 0.89 & I & 0.96 & 1 & 0.94 & 1 \\
\hline $\operatorname{PC}(16: 1 / 14: 0)$ & 704.5225 & 9.56 & $6.52 \mathrm{E}-04$ & 0.85 & $*$ & 0.93 & l & 0.96 & I & 0.98 & / & 1.10 & / \\
\hline $\operatorname{PC}(14: 0 / 18: 2)$ & 730.5381 & 9.71 & $9.24 \mathrm{E}-04$ & 0.87 & * & 0.98 & I & 1.00 & I & 1.08 & 1 & 1.13 & \# \\
\hline $\operatorname{PC}(22: 0 / 0: 0)$ & 580.4337 & 8.27 & $1.98 \mathrm{E}-03$ & 1.22 & I & 1.67 & \#\#\# & 1.44 & \#\#\# & 1.79 & \#\#\# & 1.26 & $\#$ \\
\hline $\operatorname{PC}(24: 0 / 0: 0)$ & 608.4650 & 9.35 & $3.94 \mathrm{E}-03$ & 1.29 & * & 1.55 & \#\#\# & 1.33 & \#\# & 1.60 & \#\#\# & 1.33 & \#\# \\
\hline $\operatorname{PC}(20: 0 / 0: 0)$ & 552.4024 & 7.05 & 4.50E- 03 & 1.15 & ' & 2.10 & \#\#\#\# & 1.80 & \#\#\#\# & 2.24 & \#\#\# & 1.31 & I \\
\hline PC(P-18:0/22:G) & 818.6058 & 11.36 & $2.09 \mathrm{E}-02$ & 0.91 & * & 0.89 & I & 0.91 & 1 & 0.85 & \#\# & 0.98 & 1 \\
\hline $\operatorname{PE}(18: 2 / 16: 0)$ & 716.5225 & 11.22 & $5.36 \mathrm{E}-06$ & 1.16 & I & 1.04 & I & 1.16 & I & 1.32 & \#\#\# & 1.00 & I \\
\hline $\operatorname{PE}(18: 2 / 18: 0)$ & 744.5538 & 12.66 & 7.49E-05 & 1.11 & * & 0.95 & / & 1.01 & I & 1.11 & \#\# & 1.08 & 1 \\
\hline $\operatorname{PE}(20: 4 / 16: 0)$ & 740.5225 & 11.17 & $1.01 \mathrm{E}-04$ & 1.19 & $* * *$ & 1.03 & ' & 1.09 & I & 1.26 & \#\#\# & 0.99 & / \\
\hline $\operatorname{PE}(17: 0 / 17: 0)$ & 720.5538 & 11.11 & $6.30 \mathrm{E}-03$ & 1.16 & * & 1.06 & / & 1.15 & I & 1.14 & I & 1.04 & / \\
\hline $\operatorname{PE}(18: 0 / 22: 6)$ & 792.5538 & 12.25 & $1.04 \mathrm{E}-02$ & 1.11 & * & 0.95 & I & 1.02 & 1 & 1.07 & I & 1.10 & 1 \\
\hline GlcCer(d18:1/24:0) & 812.6974 & 15.66 & $1.89 \mathrm{E}-05$ & 1.23 & $* * * *$ & 0.85 & \#\# & 0.81 & \#\#\# & 0.95 & I & 0.88 & \# \\
\hline GlcCer(d18:1/22:0) & 784.6661 & 15.17 & $8.41 \mathrm{E}-05$ & 1.17 & $* * * *$ & 0.85 & \#\#\# & 0.83 & \#\#\# & 1.00 & I & 0.84 & \#\#\# \\
\hline GlcCer(d18:1/24:1) & 810.6817 & 15.14 & $1.36 \mathrm{E}-04$ & 1.15 & $* *$ & 1.08 & / & 1.03 & \#\#\#\# & 1.24 & / & 1.06 & / \\
\hline $\operatorname{Cer}(t 18: 0 / 26: 0)$ & 696.6864 & 16.21 & $1.51 \mathrm{E}-03$ & 1.26 & $* * *$ & 1.09 & I & 1.14 & I & 1.28 & \#\#\#\# & 1.07 & 1 \\
\hline Cer(d18:1/26:0) & 678.6759 & 16.35 & $5.62 \mathrm{E}-03$ & 1.30 & *** & 0.95 & I & 0.93 & I & 0.98 & / & 1.00 & I \\
\hline Cer(d18:1/24:1) & 810.6817 & 15.14 & $2.22 \mathrm{E}-02$ & 1.16 & 1 & 1.47 & \#\#\#\# & 1.46 & \#\#\#\# & 1.28 & \# & 1.35 & \#\#\# \\
\hline $\operatorname{Cer}(\mathbf{d 1 8 : 1 / 2 2 : 0 )}$ & 784.6661 & 15.75 & $2.84 \mathrm{E}-02$ & 1.11 & I & 1.20 & $\# \#$ & 1.17 & $\#$ & 1.06 & / & 0.99 & I \\
\hline $\operatorname{SM}(\mathrm{d} 18: 1 / 24: 1)$ & 813.6844 & 14.85 & $1.88 \mathrm{E}-05$ & 1.20 & *** & 0.84 & \#\# & 0.85 & \#\#\# & 0.98 & I & 0.95 & I \\
\hline $\operatorname{SM}(\mathbf{d} 18: 1 / 26: 1)$ & 841.7157 & 15.51 & $5.70 \mathrm{E}-04$ & 1.24 & ** & 0.51 & \#\#\#\# & 0.56 & \#\#\#\# & 0.75 & \#\# & 0.71 & \#\#\# \\
\hline $\operatorname{SM}(\mathrm{d} 18: 1 / 16: 0)$ & 703.5749 & 10.49 & $1.78 \mathrm{E}-03$ & 1.12 & $* *$ & 0.77 & \#\#\# & 0.84 & \#\#\# & 0.93 & I & 0.76 & \#\#\# \\
\hline SM(d18:1/18:0) & 731.6062 & 11.81 & $2.66 \mathrm{E}-03$ & 1.15 & $* *$ & 0.72 & \#\#\#\# & 0.80 & \#\#\#\# & 0.92 & I & 0.76 & \#\#\# \\
\hline $\operatorname{SM}(\mathrm{d} 16: 1 / 18: 1)$ & 701.5592 & 9.55 & $3.45 \mathrm{E}-03$ & 0.86 & * & 0.73 & \#\#\# & 0.71 & \#\#\#\# & 0.80 & \#\# & 0.88 & I \\
\hline $\operatorname{SM}(\mathbf{d} 18: 1 / 17: 0)$ & 717.5905 & 11.11 & $9.84 \mathrm{E}-03$ & 1.10 & 1 & 0.78 & \#\# & 0.86 & \# & 0.93 & I & 0.72 & \#\#\# \\
\hline $\operatorname{SM}(\mathrm{d} 16: 1 / 24: 1)$ & 785.6531 & 13.85 & $1.66 \mathrm{E}-02$ & 0.88 & 1 & 0.87 & I & 0.89 & 1 & 0.79 & I & 0.97 & 1 \\
\hline
\end{tabular}

Colour coded according to the fold change, $n=8$; Colour bar: $\frac{\square}{\text { Low } 1 \mathrm{High}}$; $t_{R}$ : time retention; Actua M: actual mass; All the results are expressed as mean \pm SEM; All data were analysed by one-way ANOVA with Dunnett-test; ${ }^{*} P<0.05,{ }^{* *} P<0.01,{ }^{* * *} P<0.001,{ }^{* * * *} P<0.0001$ (comparing with the WT group), ${ }^{\#} P<0.05$, ${ }^{\# \#} P<0.01$, $\# \# P<0.001, \# \# \# P<0.0001$ (comparing with the Tg group) 


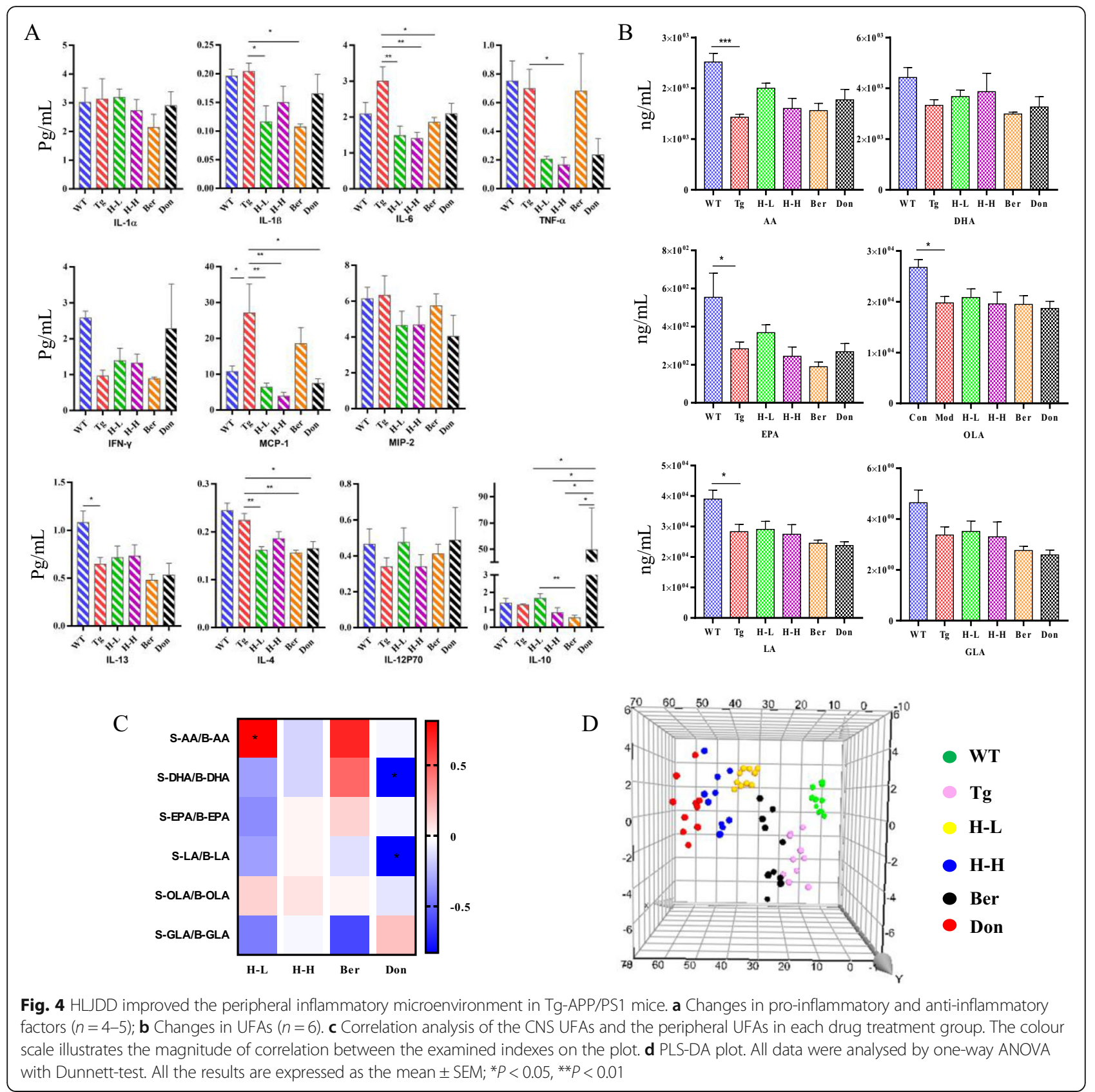

Tg mice $(0.13 \%, 1.28 \%, 0.36 \%$ respectively) than those in WT mice $(0.5 \%, 2.35 \%, 0.69 \%$ respectively), while the abundances of Lachnospiraceae_NK4A136_ group (1.5fold), Bacteroides (5.4-fold) and Odoribacte (1.3-fold) were higher. However, treatment of $\mathrm{Tg}$ mice with HLJDD increased Prevotellaceae_UCG_001, Lactobacillus, Helicobacter, Lachnospiraceae-UCG-001, Tyzzerella3, Ruminococcaceae-UCG-014 and Parasutterella, and reduced the relative abundances of $g$-unidentified, Lachnospiraceae_NK4A136_group, Bacteroides, Roseburia, Anaerotruncu, Lachnospiraceae_FCSO20_group and Odoribacte. In addition, LEfSe was used to identify the specific altered bacterial phenotype. A total of 44 bacteria changed significantly among the six groups, with a linear discriminant analysis (LDA) score $\log 10>3$ (Fig. S2).

\section{Phenotypic features of Tg-APP/PS1 mice after administration of Ber}

In general, Ber was not as effective as HLJDD in ameliorating cognitive deficiency in $\mathrm{Tg}$ mice, which was reflected in its poor performance in the MWM task and more $A \beta$ plaques in the CNS. In terms of the therapeutic mechanism, the differences between 
Table 3 The metabolic changes of 13 potential lipids markers in the periphery of $\mathrm{Tg}$ mice

\begin{tabular}{|c|c|c|c|c|c|c|c|c|c|c|c|c|c|}
\hline Group & $\begin{array}{l}\text { Actua } \\
\text { M }\end{array}$ & TR & $\begin{array}{l}\text { T-test } \\
\text { P-value }\end{array}$ & $\begin{array}{l}\text { WT } \\
/ \mathbf{T g}\end{array}$ & Sig & $\begin{array}{l}\text { H-L } \\
/ \mathrm{Tg}\end{array}$ & Sig & $\begin{array}{l}\text { H-H } \\
/ \mathrm{Tg}\end{array}$ & Sig & $\begin{array}{l}\text { Ber } \\
/ \mathrm{Tg}\end{array}$ & Sig & $\begin{array}{l}\text { Don } \\
\text { / Tg }\end{array}$ & Sig \\
\hline $\operatorname{PC}(0: 0 / 14: 0)$ & 468.31 & 1.96 & $7.43 \mathrm{E}-04$ & 1.83 & $* *$ & 2.05 & \#\#\# & 1.90 & $\# \#$ & 1.21 & I & 1.88 & \#\# \\
\hline $\operatorname{PC}(14: 0 / 0: 0)$ & 468.31 & 2.16 & $3.90 \mathrm{E}-04$ & 1.87 & $* *$ & 2.01 & \#\#\# & 1.81 & \#\# & 1.09 & / & 1.81 & \#\# \\
\hline $\operatorname{PC}(0: 0 / 16: 1)$ & 494.32 & 2.22 & $1.64 \mathrm{E}-03$ & 1.64 & l & 2.52 & \#\#\# & 2.17 & \#\#\# & 1.44 & / & 2.48 & \#\#\# \\
\hline $\operatorname{PC}(16: 1 / 0: 0)$ & 494.32 & 2.42 & $7.49 \mathrm{E}-04$ & 1.75 & $*$ & 2.47 & \#\#\#\# & 2.07 & \#\#\# & 1.28 & I & 2.14 & \#\#\# \\
\hline $\operatorname{PC}(17: 0 / 0: 0)$ & 510.36 & 4.28 & $2.48 \mathrm{E}-05$ & 1.81 & $* * *$ & 1.70 & \#\# & 1.46 & I & 0.94 & / & 1.27 & / \\
\hline $\operatorname{PC}(0: 0 / 18: 1)$ & 522.36 & 3.51 & $1.39 \mathrm{E}-03$ & 1.65 & I & 02 & \#\#\# & 2.76 & \#\#\# & 1.78 & I & .76 & \#\#\# \\
\hline $\operatorname{PC}(19: 0 / 0: 0)$ & 538.39 & 6.11 & $9.52 \mathrm{E}-05$ & 1.74 & $* *$ & 1.58 & $\#$ & 1.48 & I & 1.03 & / & 1.15 & / \\
\hline $\operatorname{PC}(20: 5 / 0: 0)$ & 542.32 & 2.09 & $1.57 \mathrm{E}-02$ & 1.41 & I & 1.93 & \#\#\# & 1.58 & I & 0.97 & / & 1.29 & I \\
\hline $\operatorname{PC}(22: 6 / 0: 0)$ & 568.34 & 2.59 & 7.49E-04 & 1.45 & * & 1.45 & $\#$ & 1.50 & \#\# & 1.19 & I & 1.19 & I \\
\hline $\operatorname{PC}(0: 0 / 22: 4)$ & 572.37 & 3.59 & $6.23 \mathrm{E}-04$ & 1.41 & I & 2.09 & \#\#\# & 1.60 & $\#$ & 1.49 & $\#$ & 1.77 & \#\#\# \\
\hline $\operatorname{PC}(20: 4 / 18: 0)$ & 810.60 & 11.84 & $5.14 \mathrm{E}-03$ & 0.74 & $*$ & 0.70 & \#\# & 0.75 & $\#$ & 1.21 & / & 0.82 & l \\
\hline PE(18:2/0:0) & 478.29 & 2.92 & $1.19 \mathrm{E}-03$ & 1.74 & $*$ & 1.89 & \#\# & 2.05 & \#\# & 1.01 & / & 1.43 & I \\
\hline GlcCer(d18:1/24:1) & 810.68 & 14.97 & $5.61 \mathrm{E}-03$ & 1.42 & * & 1.52 & \#\# & 1.37 & \# & 1.30 & I & 1.43 & \# \\
\hline
\end{tabular}

Colour coded according to the fold change, $n=10$; Colour bar: $\frac{\square \square}{\text { Low } 1 \text { High }}$; $t_{R}$ : time retention; Actua M: actual mass; All the results are expressed as mean \pm SEM; All data were analysed by one-way ANOVA with Dunnett-test; ${ }^{*} P<0.05,{ }^{* *} P<0.01,{ }^{* * *} P<0.001,{ }^{* * * *} P<0.0001$ (comparing with the WT group), ${ }^{\#} P<0.05$, ${ }^{\# \#} P<0.01$,

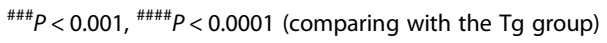

Table 4 Relative abundance of the top 20 bacteria family among six groups

\begin{tabular}{|c|c|c|c|c|c|c|c|c|c|c|c|}
\hline & \multicolumn{6}{|c|}{ Relative abundance } & \multicolumn{5}{|c|}{ Fold Change } \\
\hline & WT $\% \pm$ SEM & Tg $\% \pm$ SEM & H-L $\% \pm$ SEM & H-H $\% \pm$ SEM & Ber $\% \pm$ SEM & Don $\% \pm$ SEM & $\begin{array}{l}\text { WT } \\
/ \mathrm{Tg}\end{array}$ & $\begin{array}{c}\text { H-L } \\
/ \mathrm{Tg}\end{array}$ & $\begin{array}{c}\mathrm{H}-\mathrm{H} \\
/ \mathrm{Tg}\end{array}$ & $\begin{array}{l}\text { Ber } \\
/ \mathbf{T g}\end{array}$ & $\begin{array}{c}\text { Don } \\
/ \mathrm{Tg}\end{array}$ \\
\hline f_Clostridiaceae_1 & $9.35 \mathrm{E}-2 \pm 4.09 \mathrm{E}-2$ & $8.68 \mathrm{E}-2 \pm 2.41 \mathrm{E}-2$ & $1.69 \mathrm{E}-1 \pm 7.08 \mathrm{E}-2$ & $1.37 \mathrm{E}-1 \pm 3.26 \mathrm{E}-2$ & $3.93 \mathrm{E}-2 \pm 2.14 \mathrm{E}-2$ & $2.13 \mathrm{E}-1 \pm 8.62 \mathrm{E}-2$ & 1.08 & 1.95 & 1.58 & 0.45 & 2.45 \\
\hline f_Deferribacteraceae & $1.85 \mathrm{E}-1 \pm 1.26 \mathrm{E}-1$ & $3.20 \mathrm{E}-1 \pm 2.14 \mathrm{E}-1$ & $1.21 \mathrm{E}-1 \pm 3.83 \mathrm{E}-2$ & $8.52 \mathrm{E}-2 \pm 6.25 \mathrm{E}-2$ & $8.16 \mathrm{E}-2 \pm 4.87 \mathrm{E}-2$ & $1.31 \mathrm{E}-1 \pm 2.25 \mathrm{E}-2$ & 0.58 & 0.38 & 0.27 & 0.26 & 0.41 \\
\hline f_Unknown_Family & $2.69 \mathrm{E}-2 \pm 1.20 \mathrm{E}-2$ & $1.14 \mathrm{E}-2 \pm 4.40 \mathrm{E}-3$ & $5.96 \mathrm{E}-1 \pm 2.66 \mathrm{E}-1$ & $1.27 \mathrm{E}-1 \pm 7.59 \mathrm{E}-2$ & $5.44 \mathrm{E}-1 \pm 3.06 \mathrm{E}-1$ & $1.53 \mathrm{E}-1 \pm 8.13 \mathrm{E}-2$ & 2.36 & 52.49 & 11.18 & 47.86 & 13.50 \\
\hline f_Coriobacteriaceae & $2.90 \mathrm{E}-1 \pm 9.80 \mathrm{E}-2$ & $1.25 \mathrm{E}-1 \pm 4.29 \mathrm{E}-2$ & $3.65 \mathrm{E}-1 \pm 5.85 \mathrm{E}-2$ & $3.18 \mathrm{E}-1 \pm 7.19 \mathrm{E}-2$ & $3.80 \mathrm{E}-1 \pm 1.77 \mathrm{E}-1$ & $2.44 \mathrm{E}-1 \pm 6.96 \mathrm{E}-2$ & 2.31 & 2.91 & 2.53 & 3.02 & 1.94 \\
\hline f_unidentified & $6.25 \mathrm{E}-1 \pm 2.84 \mathrm{E}-1$ & $9.09 \mathrm{E}-2 \pm 2.15 \mathrm{E}-2$ & $2.02 \mathrm{E}-1 \pm 6.39 \mathrm{E}-2$ & $4.62 \mathrm{E}-1 \pm 3.73 \mathrm{E}-1$ & $2.27 \mathrm{E}-1 \pm 8.33 \mathrm{E}-2$ & $1.68 \mathrm{E}-1 \pm 5.05 \mathrm{E}-2$ & 6.88 & 2.23 & 5.09 & 2.49 & 1.85 \\
\hline f_Mycoplasmataceae & $1.61 \mathrm{E}-1 \pm 1.04 \mathrm{E}-1$ & $1.28 \mathrm{E}-1 \pm 3.94 \mathrm{E}-2$ & $6.01 \mathrm{E}-1 \pm 2.13 \mathrm{E}-1$ & $3.30 \mathrm{E}-1 \pm 1.70 \mathrm{E}-1$ & $8.79 \mathrm{E}-1 \pm 4.12 \mathrm{E}-1$ & $5.53 \mathrm{E}-2+5.19 \mathrm{E}-2$ & 1.26 & 4.69 & 2.58 & 6.87 & 0.43 \\
\hline f_Erysipelotrichaceae & $5.86 \mathrm{E}-1 \pm 1.56 \mathrm{E}-1$ & $2.84 \mathrm{E}-1 \pm 7.71 \mathrm{E}-2$ & $3.59 \mathrm{E}-1 \pm 1.17 \mathrm{E}-1$ & $8.15 \mathrm{E}-1 \pm 3.17 \mathrm{E}-1$ & $6.97 \mathrm{E}-1 \pm 4.19 \mathrm{E}-1$ & $3.15 \mathrm{E}-1 \pm 8.55 \mathrm{E}-2$ & 2.06 & 1.26 & 2.87 & 2.45 & 1.11 \\
\hline f_Peptococcaceae & $1.52 \mathrm{E}-1 \pm 5.67 \mathrm{E}-2$ & $9.66 \mathrm{E}-2 \pm 1.70 \mathrm{E}-2$ & $6.31 \mathrm{E}-1 \pm 1.11 \mathrm{E}-1$ & $7.87 \mathrm{E}-1 \pm 1.88 \mathrm{E}-1$ & $2.92 \mathrm{E}-1 \pm 1.40 \mathrm{E}-1$ & $1.10 \mathrm{E}+0 \pm 1.39 \mathrm{E}-1$ & 1.57 & 6.53 & 8.14 & 3.02 & 11.39 \\
\hline f_Desulfovibrionaceae & $3.85 \mathrm{E}-1 \pm 1.02 \mathrm{E}-1$ & $6.48 \mathrm{E}-1 \pm 1.59 \mathrm{E}-1$ & $7.01 \mathrm{E}-1 \pm 1.69 \mathrm{E}-1$ & $3.68 \mathrm{E}-1 \pm 7.21 \mathrm{E}-2$ & $5.04 \mathrm{E}-1 \pm 1.08 \mathrm{E}-1$ & $7.13 \mathrm{E}-1 \pm 1.12 \mathrm{E}-1$ & 0.59 & 1.08 & 0.57 & 0.78 & 1.10 \\
\hline f_Alcaligenaceae & $5.08 \mathrm{E}-1 \pm 1.86 \mathrm{E}-1$ & $1.58 \mathrm{E}-1 \pm 5.10 \mathrm{E}-2$ & $6.37 \mathrm{E}-1 \pm 1.41 \mathrm{E}-1$ & $1.01 \mathrm{E}+0 \pm 2.29 \mathrm{E}-1$ & $2.47 \mathrm{E}-1 \pm 5.17 \mathrm{E}-2$ & $8.11 \mathrm{E}-1 \pm 2.87 \mathrm{E}-1$ & 3.21 & 4.03 & 6.39 & 1.57 & 5.13 \\
\hline f_Porphyromonadaceae & $7.92 \mathrm{E}-1 \pm 2.27 \mathrm{E}-1$ & $1.77 \mathrm{E}+0 \pm 3.31 \mathrm{E}-1$ & $8.09 \mathrm{E}-1 \pm 2.25 \mathrm{E}-1$ & $5.74 \mathrm{E}-1 \pm 1.22 \mathrm{E}-1$ & $3.22 \mathrm{E}-1 \pm 9.44 \mathrm{E}-2$ & $6.71 \mathrm{E}-1 \pm 9.12 \mathrm{E}-2$ & 0.45 & 0.46 & 0.32 & 0.18 & 0.38 \\
\hline f_Rikenellaceae & $1.20 \mathrm{E}+0 \pm 3.63 \mathrm{E}-1$ & $1.56 \mathrm{E}+0 \pm 3.33 \mathrm{E}-1$ & $9.75 \mathrm{E}-1 \pm 2.82 \mathrm{E}-1$ & $1.13 \mathrm{E}+0 \pm 3.58 \mathrm{E}-1$ & $8.25 \mathrm{E}-1 \pm 4.75 \mathrm{E}-1$ & $1.74 \mathrm{E}+0 \pm 6.67 \mathrm{E}-1$ & 0.77 & 0.63 & 0.73 & 0.53 & 1.12 \\
\hline f_Bacteroidaceae & $8.81 \mathrm{E}-1 \pm 2.50 \mathrm{E}-1$ & $4.77 \mathrm{E}+0 \pm 1.24 \mathrm{E}+0$ & $2.75 \mathrm{E}+0 \pm 6.80 \mathrm{E}-1$ & $2.89 \mathrm{E}+0 \pm 9.73 \mathrm{E}-1$ & $1.39 \mathrm{E}+1 \pm 4.51 \mathrm{E}+0$ & $3.16 \mathrm{E}+0 \pm 5.21 \mathrm{E}-1$ & 0.18 & 0.58 & 0.61 & 2.92 & 0.66 \\
\hline f_Helicobacteraceae & $1.67 \mathrm{E}+0 \pm 5.89 \mathrm{E}-1$ & $2.13 \mathrm{E}+0 \pm 6.20 \mathrm{E}-1$ & $8.57 \mathrm{E}+0 \pm 2.79 \mathrm{E}+0$ & $6.16 \mathrm{E}+0 \pm 2.59 \mathrm{E}+0$ & $2.36 \mathrm{E}+0 \pm 9.67 \mathrm{E}-1$ & $8.12 \mathrm{E}+0 \pm 1.13 \mathrm{E}+0$ & 0.78 & 4.02 & 2.88 & 1.10 & 3.80 \\
\hline f_Lactobacillaceae & $2.74 \mathrm{E}+0 \pm 1.03 \mathrm{E}+0$ & $2.63 \mathrm{E}+0 \pm 1.06 \mathrm{E}+0$ & $1.22 \mathrm{E}+1 \pm 3.59 \mathrm{E}+0$ & $4.87 \mathrm{E}+0 \pm 1.46 \mathrm{E}+0$ & $1.04 \mathrm{E}+0 \pm 2.53 \mathrm{E}-1$ & $6.11 \mathrm{E}+0 \pm 1.92 \mathrm{E}+0$ & 1.05 & 4.65 & 1.86 & 0.39 & 2.33 \\
\hline f_Ruminococcaceae & $4.01 \mathrm{E}+0 \pm 9.32 \mathrm{E}-1$ & $4.52 \mathrm{E}+0 \pm 7.33 \mathrm{E}-1$ & $5.14 \mathrm{E}+0 \pm 9.80 \mathrm{E}-1$ & $3.77 \mathrm{E}+0 \pm 6.05 \mathrm{E}-1$ & $6.93 \mathrm{E}+0 \pm 1.30 \mathrm{E}+0$ & $5.46 \mathrm{E}+0 \pm 8.51 \mathrm{E}-1$ & 0.89 & 1.14 & 0.83 & 1.53 & 1.21 \\
\hline f_Prevotellaceae & $3.72 \mathrm{E}+0 \pm 9.02 \mathrm{E}-1$ & $4.85 \mathrm{E}+0 \pm 1.80 \mathrm{E}+0$ & $1.39 \mathrm{E}+1 \pm 2.76 \mathrm{E}+0$ & $1.38 \mathrm{E}+1 \pm 3.32 \mathrm{E}-+0$ & $1.27 \mathrm{E}-1 \pm 1.26 \mathrm{E}-1$ & $1.08 \mathrm{E}+1 \pm 1.52 \mathrm{E}+0$ & 0.77 & 2.87 & 2.84 & 0.03 & 2.23 \\
\hline f_Lachnospiraceae & $2.72 \mathrm{E}+1 \pm 6.71 \mathrm{E}+0$ & $3.39 \mathrm{E}+1 \pm 4.99 \mathrm{E}+0$ & $2.39 \mathrm{E}+1 \pm 4.66 \mathrm{E}+0$ & $2.91 \mathrm{E}+1 \pm 4.99 \mathrm{E}+0$ & $1.53 \mathrm{E}+1 \pm 2.71 \mathrm{E}+0$ & $3.18 \mathrm{E}+1 \pm 3.41 \mathrm{E}+0$ & 0.80 & 0.70 & 0.86 & 0.45 & 0.94 \\
\hline f_Bacteroidales_S24-7_group & $5.37 \mathrm{E}+1 \pm 7.53 \mathrm{E}+0$ & $4.13 \mathrm{E}+1 \pm 5.51 \mathrm{E}+0$ & $2.66 \mathrm{E}+1 \pm 4.43 \mathrm{E}+0$ & $3.28 \mathrm{E}+1 \pm 2.85 \mathrm{E}+0$ & $5.40 \mathrm{E}+1 \pm 4.15 \mathrm{E}+0$ & $2.77 \mathrm{E}+1 \pm 4.28 \mathrm{E}+0$ & 1.30 & 0.64 & 0.79 & 1.31 & 0.67 \\
\hline f_Rickettsiales_Incertae_Sedis & $4.13 \mathrm{E}-2 \pm 2.73 \mathrm{E}-2$ & $\mathrm{ND}$ & $1.80 \mathrm{E}-1 \pm 9.50 \mathrm{E}-2$ & $1.41 \mathrm{E}-1 \pm 4.03 \mathrm{E}-2$ & $2.39 \mathrm{E}-1 \pm 1.72 \mathrm{E}-1$ & $2.40 \mathrm{E}-1 \pm 4.45 \mathrm{E}-2$ & & & & & \\
\hline
\end{tabular}

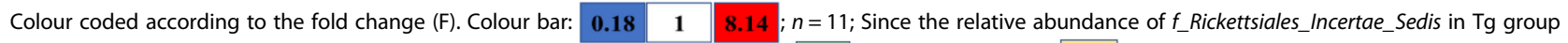
was zero, its fold change between groups were not calculated; ND: not detected; $\square$ : Less than 10 times; :more than 10 times; All the results are expressed as mean \pm SEM 
Table 5 Relative abundance of the top 20 bacteria genus among six groups

\begin{tabular}{|c|c|c|c|c|c|c|c|c|c|c|c|}
\hline & \multicolumn{6}{|c|}{ Relative abundance } & \multicolumn{5}{|c|}{ Fold change } \\
\hline & WT $\% \pm$ SEM & Tg $\% \pm$ SEM & H-L $\% \pm$ SEM & H-H $\% \pm$ SEM & Ber $\% \pm$ SEM & Don $\% \pm$ SEM & $\begin{array}{l}\text { WT } \\
/ \text { Tg }\end{array}$ & $\begin{array}{c}\text { H-L } \\
/ \mathrm{Tg}\end{array}$ & $\begin{array}{c}\mathrm{H}-\mathrm{H} \\
/ \mathrm{Tg}\end{array}$ & $\begin{array}{l}\text { Ber } \\
/ \mathrm{Tg}\end{array}$ & $\begin{array}{l}\text { Don } \\
/ \mathbf{T g}\end{array}$ \\
\hline g_Odoribacter & $7.34 \mathrm{E}-1 \pm 2.31 \mathrm{E}-1$ & $1.42 \mathrm{E}+0 \pm 3.66 \mathrm{E}-1$ & $3.40 \mathrm{E}-1 \pm 1.28 \mathrm{E}-1$ & $2.23 \mathrm{E}-1 \pm 9.96 \mathrm{E}-2$ & $3.56 \mathrm{E}-2 \pm 1.96 \mathrm{E}-2$ & $3.35 \mathrm{E}-1 \pm 8.69 \mathrm{E}-2$ & 0.52 & 0.24 & 0.16 & 0.03 & 0.24 \\
\hline g_Desulfovibrio & $3.68 \mathrm{E}-1 \pm 1.00 \mathrm{E}-1$ & $6.29 \mathrm{E}-1 \pm 1.53 \mathrm{E}-1$ & $6.42 \mathrm{E}-1 \pm 1.63 \mathrm{E}-1$ & $3.41 \mathrm{E}-1 \pm 6.65 \mathrm{E}-2$ & $4.83 \mathrm{E}-1 \pm 1.02 \mathrm{E}-1$ & $6.52 \mathrm{E}-1 \pm 1.03 \mathrm{E}-1$ & 0.59 & 1.02 & 0.54 & 0.77 & 1.04 \\
\hline g_Lachnoclostridium & $5.20 \mathrm{E}-1 \pm 1.27 \mathrm{E}-1$ & $5.40 \mathrm{E}-1 \pm 1.51 \mathrm{E}-1$ & $3.41 \mathrm{E}-1 \pm 4.74 \mathrm{E}-2$ & $5.16 \mathrm{E}-1 \pm 1.59 \mathrm{E}-1$ & $9.59 \mathrm{E}-1 \pm 2.45 \mathrm{E}-1$ & $3.63 \mathrm{E}-1 \pm 6.64 \mathrm{E}-2$ & 0.96 & 0.63 & 0.96 & 1.77 & 0.67 \\
\hline g_mouse_gut_metagenome & $7.34 \mathrm{E}-1 \pm 2.68 \mathrm{E}-1$ & $8.17 \mathrm{E}-1 \pm 2.35 \mathrm{E}-1$ & $3.51 \mathrm{E}-1 \pm 1.38 \mathrm{E}-1$ & $7.87 \mathrm{E}-1 \pm 1.06 \mathrm{E}-1$ & $8.26 \mathrm{E}-3 \pm 4.36 \mathrm{E}-3$ & $6.09 \mathrm{E}-1 \pm 1.41 \mathrm{E}-1$ & 0.90 & 0.43 & 0.96 & 0.01 & 0.75 \\
\hline g_Parasutterella & $5.04 \mathrm{E}-1 \pm 1.86 \mathrm{E}-1$ & $1.31 \mathrm{E}-1 \pm 5.09 \mathrm{E}-2$ & $6.35 \mathrm{E}-1 \pm 1.40 \mathrm{E}-1$ & $1.01 \mathrm{E}+0 \pm 2.29 \mathrm{E}-1$ & $2.34 \mathrm{E}-1 \pm 5.25 \mathrm{E}-2$ & $8.08 \mathrm{E}-1 \pm 2.87 \mathrm{E}-1$ & 3.85 & 4.86 & 7.72 & 1.79 & 6.19 \\
\hline g_Ruminococcaceae_UCG-014 & $6.86 \mathrm{E}-1 \pm 1.70 \mathrm{E}-1$ & $3.55 \mathrm{E}-1 \pm 8.53 \mathrm{E}-2$ & $9.18 \mathrm{E}-1 \pm 2.71 \mathrm{E}-1$ & $5.41 \mathrm{E}-1 \pm 1.37 \mathrm{E}-1$ & $5.42 \mathrm{E}-1 \pm 2.18 \mathrm{E}-1$ & $4.08 \mathrm{E}-1 \pm 1.77 \mathrm{E}-1$ & 1.93 & 2.59 & 1.53 & 1.53 & 1.15 \\
\hline g_Tyzzerella_3 & $3.46 \mathrm{E}-2 \pm 1.57 \mathrm{E}-2$ & $4.13 \mathrm{E}-3 \pm 3.60 \mathrm{E}-3$ & $9.40 \mathrm{E}-2 \pm 4.69 \mathrm{E}-2$ & $1.78 \mathrm{E}+0 \pm 3.88 \mathrm{E}-1$ & $0.00 \mathrm{E}+0 \pm 0.00 \mathrm{E}+0$ & $1.76 \mathrm{E}+0 \pm 9.60 \mathrm{E}-1$ & 8.38 & 22.75 & 431.25 & 0.00 & 426.13 \\
\hline g_Alistipes & $7.48 \mathrm{E}-1 \pm 3.03 \mathrm{E}-1$ & $8.13 \mathrm{E}-1 \pm 2.64 \mathrm{E}-1$ & $5.43 \mathrm{E}-1 \pm 2.14 \mathrm{E}-1$ & $4.31 \mathrm{E}-1 \pm 1.12 \mathrm{E}-1$ & $7.65 \mathrm{E}-1 \pm 4.59 \mathrm{E}-1$ & $4.54 \mathrm{E}-1 \pm 9.17 \mathrm{E}-2$ & 0.92 & 0.67 & 0.53 & 0.94 & 0.56 \\
\hline g_Lachnospiraceae_FCS020_group & $7.63 \mathrm{E}-1 \pm 2.49 \mathrm{E}-1$ & $8.65 \mathrm{E}-1 \pm 4.21 \mathrm{E}-1$ & $3.75 \mathrm{E}-1 \pm 8.66 \mathrm{E}-2$ & $6.38 \mathrm{E}-1 \pm 1.38 \mathrm{E}-1$ & $6.29 \mathrm{E}-1 \pm 1.28 \mathrm{E}-1$ & $5.69 \mathrm{E}-1 \pm 2.10 \mathrm{E}-1$ & 0.88 & 0.43 & 0.74 & 0.73 & 0.66 \\
\hline g_Ruminiclostridium_9 & $2.86 \mathrm{E}-1 \pm 7.10 \mathrm{E}-2$ & $5.49 \mathrm{E}-1 \pm 1.57 \mathrm{E}-1$ & $8.68 \mathrm{E}-1 \pm 2.38 \mathrm{E}-1$ & $6.03 \mathrm{E}-1 \pm 1.04 \mathrm{E}-1$ & $9.87 \mathrm{E}-1 \pm 2.86 \mathrm{E}-1$ & $9.60 \mathrm{E}-1 \pm 1.80 \mathrm{E}-1$ & 0.52 & 1.58 & 1.10 & 1.80 & 1.75 \\
\hline g_Alloprevotella & $7.64 \mathrm{E}-2 \pm 2.65 \mathrm{E}-2$ & $8.29 \mathrm{E}-1 \pm 3.47 \mathrm{E}+0$ & $1.57 \mathrm{E}+0 \pm 6.44 \mathrm{E}-1$ & $1.11 \mathrm{E}+0 \pm 5.56 \mathrm{E}-1$ & $0.00 \mathrm{E}+0 \pm 0.00 \mathrm{E}+0$ & $9.44 \mathrm{E}-1 \pm 4.14 \mathrm{E}-1$ & 0.09 & 1.89 & 1.34 & 0.00 & 1.14 \\
\hline $\mathrm{g} \quad$ Anaerotruncus & $6.07 \mathrm{E}-1 \pm 1.82 \mathrm{E}-1$ & $1.03 \mathrm{E}+0 \pm 4.69 \mathrm{E}-1$ & $7.12 \mathrm{E}-1 \pm 1.88 \mathrm{E}-1$ & 4.73E- $1 \pm 7.90 \mathrm{E}-2$ & $9.01 \mathrm{E}-1 \pm 1.52 \mathrm{E}-1$ & $9.39 \mathrm{E}-1 \pm 1.69 \mathrm{E}-1$ & 0.59 & 0.69 & 0.46 & 0.88 & 0.92 \\
\hline g_Roseburia & $2.22 \mathrm{E}+0 \pm 7.61 \mathrm{E}-1$ & $2.53 \mathrm{E}+0 \pm 1.12 \mathrm{E}+0$ & $1.72 \mathrm{E}+0 \pm 6.06 \mathrm{E}-1$ & $1.22 \mathrm{E}+0 \pm 4.88 \mathrm{E}-1$ & $1.34 \mathrm{E}+0 \pm 2.54 \mathrm{E}-1$ & $1.35 \mathrm{E}+0 \pm 3.21 \mathrm{E}-1$ & 0.88 & 0.68 & 0.48 & 0.53 & 0.53 \\
\hline g_Lachnospiraceae_UCG-001 & $2.35 \mathrm{E}+0 \pm 9.17 \mathrm{E}-1$ & $1.28 \mathrm{E}+0 \pm 6.88 \mathrm{E}-1$ & $5.22 \mathrm{E}+0 \pm 2.09 \mathrm{E}+0$ & $2.28 \mathrm{E}+0 \pm 7.43 \mathrm{E}-1$ & $1.12 \mathrm{E}-1 \pm 7.38 \mathrm{E}-2$ & $5.43 \mathrm{E}+0 \pm 1.21 \mathrm{E}+0$ & 1.84 & 4.08 & 1.78 & 0.09 & 4.24 \\
\hline g_Bacteroides & $8.81 \mathrm{E}-1 \pm 2.50 \mathrm{E}-1$ & $4.77 \mathrm{E}+0 \pm 1.24 \mathrm{E}+0$ & $2.75 \mathrm{E}+0 \pm 6.80 \mathrm{E}-1$ & $2.89 \mathrm{E}+0 \pm 9.73 \mathrm{E}-1$ & $1.39 \mathrm{E}+1 \pm 4.51 \mathrm{E}+0$ & $3.16 \mathrm{E}+0 \pm 5.21 \mathrm{E}-1$ & 0.18 & 0.58 & 0.61 & 2.92 & 0.66 \\
\hline g_Helicobacter & $1.67 \mathrm{E}+0 \pm 5.89 \mathrm{E}-1$ & $2.13 \mathrm{E}+0 \pm 6.20 \mathrm{E}-1$ & $8.57 \mathrm{E}+0 \pm 2.79 \mathrm{E}+0$ & $6.16 \mathrm{E}+0 \pm 2.59 \mathrm{E}+0$ & $2.36 \mathrm{E}+0 \pm 9.67 \mathrm{E}-1$ & $8.12 \mathrm{E}+0 \pm 1.13 \mathrm{E}+0$ & 0.78 & 4.02 & 2.88 & 1.10 & 3.80 \\
\hline g_Lactobacillus & $2.74 \mathrm{E}+0 \pm 1.03 \mathrm{E}+0$ & $2.63 \mathrm{E}+0 \pm 1.06 \mathrm{E}+0$ & $1.22 \mathrm{E}+1 \pm 3.59 \mathrm{E}+0$ & $4.87 \mathrm{E}+0 \pm 1.46 \mathrm{E}+0$ & $1.04 \mathrm{E}+0 \pm 2.53 \mathrm{E}-1$ & $6.11 \mathrm{E}+0 \pm 1.92 \mathrm{E}+0$ & 1.05 & 4.65 & 1.86 & 0.39 & 2.33 \\
\hline g_Prevotellaceae_UCG-001 & $3.42 \mathrm{E}+0 \pm 9.17 \mathrm{E}-1$ & $3.92 \mathrm{E}+0 \pm 1.55 \mathrm{E}+0$ & $1.21 \mathrm{E}+1 \pm 2.42 \mathrm{E}+0$ & $1.23 \mathrm{E}+1 \pm 3.04 \mathrm{E}+0$ & $1.27 \mathrm{E}-1 \pm 1.26 \mathrm{E}-1$ & $9.62 \mathrm{E}+0 \pm 1.41 \mathrm{E}+0$ & 0.87 & 3.08 & 3.13 & 0.03 & 2.46 \\
\hline g_Lachnospiraceae_NK4A136_group & $1.14 \mathrm{E}+1 \pm 3.67 \mathrm{E}+0$ & $1.73 \mathrm{E}+1 \pm 3.32 \mathrm{E}+0$ & $8.35 \mathrm{E}+0 \pm 2.69 \mathrm{E}+0$ & $1.72 \mathrm{E}+1 \pm 4.47 \mathrm{E}+0$ & $7.10 \mathrm{E}+0 \pm 1.84 \mathrm{E}+0$ & $1.35 \mathrm{E}+1 \pm 3.04 \mathrm{E}+0$ & 0.66 & 0.48 & 0.99 & 0.41 & 0.78 \\
\hline g_unidentified & $6.31 \mathrm{E}+1 \pm 5.91 \mathrm{E}+0$ & $5.12 \mathrm{E}+1 \pm 4.38 \mathrm{E}+0$ & $3.42 \mathrm{E}+1 \pm 3.83 \mathrm{E}+0$ & $3.85 \mathrm{E}+1 \pm 2.79 \mathrm{E}+0$ & $5.86 \mathrm{E}+1 \pm 4.32 \mathrm{E}+0$ & $3.66 \mathrm{E}+1 \pm 3.87 \mathrm{E}+0$ & 1.23 & 0.67 & 0.75 & 1.14 & 0.72 \\
\hline
\end{tabular}

Colour coded according to the fold change (F). Colour bar: $0.16 \quad 1 \quad 8.38 ; n=11 ;$ Since the relative abundance of $f \_$Rickettsiales_Incertae_Sedis in the Tg group

was zero, its fold change between groups were not calculated; $\square$ : Less than 10 times; :more than 10 times; All the results are expressed as mean \pm SEM

Ber and HLJDD were mainly manifested in the regulation of neuroinflammation, lipid metabolism, and the gut microbiota. In detail, HLJDD could upregulate a variety of neurotransmitters, which was similar to Don. Ber mainly regulated citrulline, urea, and methionine. The effects of Ber on central and peripheral inflammatory cytokines were not as good as HLJDD. But Ber significantly increased the levels of IL-13 in the brain. Combining the performance of the lipid metabolic profiling of the brain with that of serum, these plot for PLS-DA presented clear separation between HLJDD (including $\mathrm{H}-\mathrm{L}$ and $\mathrm{H}-\mathrm{H}$ group) and Ber groups (Figs. 3d, 4d). As shown in the Venn plots (Fig. S3a), PC (O-18:0/16:0) and Cer (d18:1/24:1) were the characteristic markers of the HLJDD groups in the CNS. PC (18:1/17:0), PC (16:0/18:1), PE (22:6/0:0), PE (18:0/18:1) and PE (0:0/18:1) were the characteristic markers of the HLJDD groups in the periphery. PC (18:0/18:2) was the characteristic marker of the Ber group (Fig. S3b). Moreover, we found that Ber was similar to HLJDD in regulating the CNS pathological lipids, but its effect on peripheral pathological lipids was not as good as HLJDD. Compared with HLJDD, Ber had a weaker regulatory effect on DHA, EPA and OLA. In addition, the mechanism of Ber in regulating gut microflora was different from that of HLJDD (Tables 4, 5). After Ber administration, the proportion of special enterobacterium (Prevotellaceae, Odoribacter, Tyzzerella_3, Alloprevotella, Lachnospiraceae_UCG-001 and Prevotellaceae_UCG-001) in TgAPP/PS1 mice decreased sharply. Moreover, it was found that Ber had the opposite regulatory effect compared with HLJDD on Prevotellaceae, Clostridiaceae, Bacteroidaceae, Lactobacillaceae, Bacteroidales S24-7_group, Lachnoclostridium, Tyzzerella_3, Alloprevotella, Lachnospiraceae_UCG-001, Bacteroides, Lactobacillus and Prevotellaceae_UCG-001.

\section{Discussion}

In the present study, the central neuroinflammation was closely related to the onset of AD. The disorders in LPs metabolism and intestinal flora were the potential drivers (Fig. 5). 16S rRNA gene sequencing of the gut microbiome and integrated metabolomics were adopted to monitor the phenotypic features of APP/PS1 mice. The disorder of both biochemical factors in the brain and the microbial diversity in intestine contributed to the neuroinflammation. HLJDD could reverse them and then improve the cognitive impairment in Tg mice. 
The elevation of $A \beta$ level induced the overexpression of pro-inflammatory cytokines and chemokines to robust inflammatory response [20]. The more severe inflammatory degree was found in the brain of $\mathrm{Tg}$ mice than that in WT mice, followed with $A \beta$ plaques accumulation and abnormal expression of inflammatory cytokines, such as IFN- $\gamma$, IL-12p70 and IL-6. Meanwhile, the elevated level of $A \beta_{42}$ was closely associated with the increased level of oxidative stress [60], which was mainly reflected in the decrease of SOD and the increase of methionine in Tg-APP/PS1 mice. Methionine could increase the levels of $A \beta$ and nitro-tyrosinated protein, which further induced neuroinflammation [61, 62]. In addition, the evidence showed that inflammation in $\mathrm{Tg}$ mice was not only confined to the CNS but also spread to the periphery. Compared with WT mice, both IFN- $\gamma$ and IL- 6 in the CNS and periphery of Tg mice showed significant changes. Upregulation of IL-6 was predictive of progression to $A D$, which was verified in various $A D$ models and patients with varying degrees of $\operatorname{AD}[9,63]$. Study reported that INF- $\gamma$ has dual roles in Alzheimer's disease [64]. Browne et al. reported that the release of INF- $\gamma$ from infiltrating Th1 cells plays a diabolical role in AD pathogenesis. There was still reports that INF- $\gamma$ at the brain's choroid plexus were reduced in brain ageing $[65,66]$ and under neurodegenerative condition [67]. The availability of INF- $\gamma$ at the brain of AD mice might be affected by systemic immune suppression. Meanwhile, the higher level of MCP-1 in the serum was found in Tg mice compared with WT mice, which appeared to be associated with greater severity and a faster cognitive decline $[68,69]$.

Many $A \beta$-produced proteins have been found in lipid rafts such as $A \beta$ protein precursor ( $\beta A P P), \beta$-secretase, $\gamma$ - secretase and neprilysin [70]. In addition, $A \beta$ directly disrupt bilayer integrity by interacting with PLs [71]. Data from targeted lipidomics showed that Tg mice were characterized by a decrease in PCs, PEs and SMs of the CNS or periphery. Previous evidence has shown that a reduced concentration of PCs in the CNS and peripheral system was associated with impaired cognitive performance in older individuals and $\mathrm{AD}$ patients [27, 28, 72]. Among them, PC (16:0/16:0) was predictive of progression to $\mathrm{AD}$ dementia in individuals with mild cognitive impairment (MCI) [73, 74]. Oxidative and lipid peroxidation were early events in $\mathrm{AD}$ [75]. In this study, the results of PEs, PCs and SOD were in accordance with the previous paper, which suggested the implication of oxidative stress in the progressive degradation of brain PLs in AD. PCs and PEs were rich in readily oxidizable AAs and DHAs [76, 77]. We speculated that the metabolic disorder of PC and PE induced the abnormal expression of $\mathrm{A} \beta$ by breaking down the lipid rafts homeostasis, which deteriorated neuroinflammation.
PUFAs act as precursors for biosynthesis of the lipid mediators, which are actively involved in the inflammatory response. We found that the levels of brain PUFAs had no significant difference in $\mathrm{Tg}$ mice compared to WT mice. Others have shown that the brain PUFAs differ very little in AD compared to healthy people [7880]. However, the contents of PUFAs and OLA in the periphery of $\mathrm{Tg}$ mice decreased significantly compared with those of WT mice. A higher level of AA is strongly associated with $\mathrm{AD}$ by yielding some inflammatory mediators [81, 82]. Conversely, some study still believes that a lower level of AA is associated with cognitive decline $[83,84]$. In this study, the level of AA in the periphery of Tg mice decreased compared with WT mice, which was attributed to the decline of upstream molecules, including OLA, LA and GLA. LA, GLA and OLA participate in numerous cellular functions by affecting membrane fluidity, membrane enzymatic activities and eicosanoid synthesis [84].

The present study in $\mathrm{Tg}$ mice indicated that alterations in the gut microbiome contributed to neuroinflammation. Study reported that the circulating omega-3 fatty acid (DHA, EPA) can influence the composition of the host gut flora, especially SCFA-producing bacteria [85]. SCFA-producing bacteria decreased in Tg mice compared with the WT mice, including Parasutterella and Blautia. SCFAs can interact with nerve cells by stimulating the sympathetic and autonomic nervous system [86, 87]. SCFAs even cross the BBB and regulate microglial homeostasis. A decrease of SCFA-producing bacteria aggravated cognitive decline. Porphyromonadaceae is highly associated with inflammatory diseases [88, 89], and even induces cognitive decline and anxiety-like behaviour [90]. Increases in bacterial taxa from this family have also been observed in faecal samples from individuals with major depressive disorders, especially Odoribacter [91]. An increase in intestinal Odoribacter was certified in a variety of AD models [58, 90, 92]. Likewise, we observed that the proportion of Porphyromonadaceae and Odoribacter increased in $\mathrm{Tg}$ mice compared with WT mice, which expected to be a potential biomarker to predict the occurrence of $\mathrm{AD}$.

Circulating BAs provide an important mechanism for communication between the gut and the brain [48, 93, 94]. Alterations in the gut microbiome significantly affect BAs transformation through various microbial enzymes such as bile salt hydrolase (BSHs) and hydroxysteroid dehydrogenases [95]. Compared with WT mice, an increase of tauro-conjugated BAs was observed in $\mathrm{Tg}$ APP/PS1 mice, probably a result of lower BSHs activity from Lactobacillus. Indeed, Lactobacillus have BSHs activity for catalyzing deconjugation of tauro-conjugated BAs [96]. Increased amounts of secondary BAs in the blood may enter the brain through the permeability of 


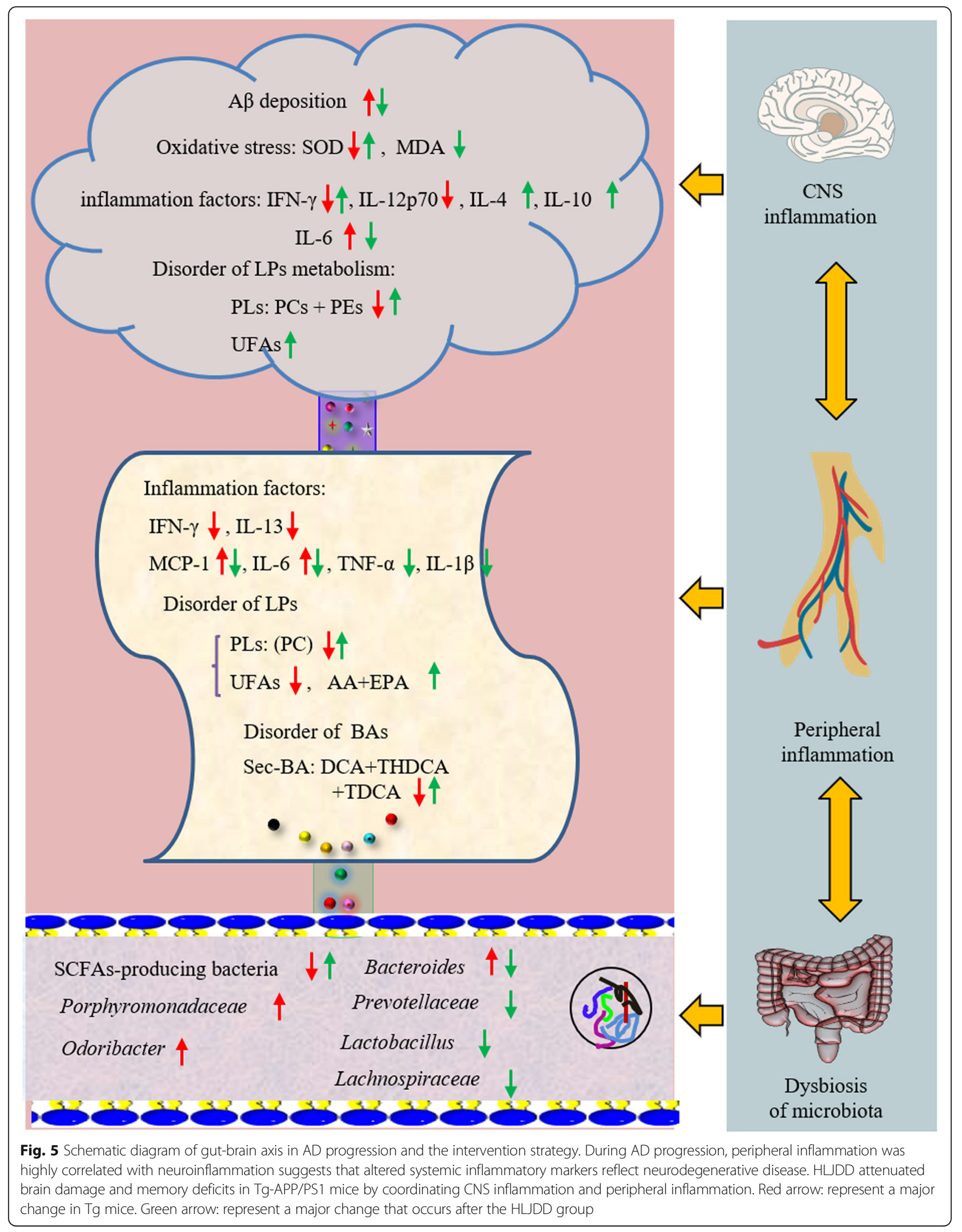


the $\mathrm{BBB}$ and affect brain physiology and metabolism [97]. DCA represent aetiological agents and mediators of the pathogenic process $[98,99]$. A higher level of DCA was significantly associated with worse cognitive performance [100]. The decline of CA level is thought to be one of the causes of cognitive impairment in $A D$ patients [47]. The decline of CA in Tg-APP/PS1 mice may be caused by species difference $[47,100]$. In addition, the ratios of secondary to primary BAs increased in $\mathrm{Tg}$ mice relative to WT mice. This indicated that the enzymatic steps in the conversion of primary to secondary BAs in the gut might contribute to disease.

The present study demonstrated that HLJDD with strict quality control could reverse cognitive impairment in $\mathrm{Tg}$ mice. It is worth mentioning that HLJDD decreased the expression of IL- 6 in both of brain and serum of Tg mice. Upregulation of IL- 6 forced microglia polarization to pro-inflammatory and neurodegeneration [101]. In addition, the regulation of HLJDD on central NTs was similar to that of Don. The previous literature reported that HLJDD ameliorates the cognitive dysfunction by regulating LP metabolism [9]. The present study indicated that HLJDD could relieve the neuroinflammation by increasing the levels of PCs, PEs, DHA and EPA. In the correlation network analysis, GLA, OLA, LA, EPA and DHA were negatively correlated at the central and peripheral levels after administration of HLJDD. However, the contents of AA in periphery showed a positive association with that in the CNS. These results suggested that HLJDD regulated the performance of central PUFAs by alleviating peripheral PUFAs, since these essential PUFAs in the brain are primarily derived from the peripheral circulation [102]. In addition, we observed that HLJDD effectively reshaped the gut microbiota structure in Tg mice. HLJDD enriched the gut microbiota SCFA-producing bacteria population. The circulating DHA was positively correlated with SCFA-producing bacteria. Therefore, we speculated that HLJDD may alter the microbiota composition by affecting the levels of DHA in Tg mice. Meanwhile, HLJDD could increase the populations of Prevotellaceae and its genus, including Prevotellaceae_UCG-001, Prevotellaceae_Ga6A1_group and Parasutterella. These bacteria were reported to be reduced in PD patients and Tg mice [103, 104]. Prevotella is known to breakdown complex carbohydrates, providing SCFAs as well as thiamine and folate as by products that promote a healthy intestinal environment. The decrease of Prevotella may reduce the levels of these important micronutrients.

The previous study demonstrated that HLJDD restored the dysregulated microbiota structure and function by increasing SCFA-producing bacteria in T2DM rats [105]. Lachnospiraceae can induce cognitive defect in $\mathrm{AD}$ patients [106] by intervening in the mucosal integrity of the host, BA, glucose and lipid metabolism [107]. In addition, HLJDD could remodel serum BAs homeostasis in $\mathrm{Tg}$ mice by decreasing the populations of Lachnospiraceae and its followed genera and increasing the populations of Bacteroides and Lactobacillus. Bacteroides and Lactobacillus were reported to be actively involved in BAs metabolism, including deconjugation, the hydroxyl groups at C3, C7 and C12, and desulfatation [108]. The ratios of secondary to primary BAs changed in Tg mice, which proved that HLJDD affected BAs metabolism by alternating microbiota structure. The ratio of secondary BAs to primary BAs is used to assess differences in the activity of intestinal microbiome enzymes that can induce BAs changes [47].

\section{Limitations}

This study had some limitations. Only MWM test was performed to assess short term memory which is impaired in AD. Actually, various behavioural assessments were indeed the best strategy for the comprehensive evaluation of memory. In addition, although we have revealed existing communication disorders of the "braingut" axis in the pathogenesis of $\mathrm{AD}$, further studies to identify the key bacterial strains and functional enzymes that may account for metabolites and neuroinflammation changes will be critical. Further research on these key issues may help gain a better understanding of the pathogenesis of $\mathrm{AD}$ and perfectly elucidate the potential mechanism of HLJDD in the treatment of AD.

\section{Conclusion}

In the present study, neuroinflammation was closely related to the onset of $\mathrm{AD}$, and disorders in LP metabolism and intestinal flora, which were the potential drivers. Peripheral inflammation displayed the crosstalk with CNS inflammation and cognitive impairment. The combination of inflammatory factors (IL-6 and INF- $\gamma$ ), PCs and SCFA-producing bacteria was a promising early diagnostic biomarker for $\mathrm{AD}$, which worths further validation in numerous experiments. HLJDD suppressed gut dysbiosis and the associated $\mathrm{A} \beta$ accumulation, harnessed neuroinflammation and reversed cognitive impairment. More importantly, the identified antiinflammatory actions of HLJDD will open a new therapeutic avenue for $\mathrm{AD}$ treatment through an intervention pattern in the "brain-gut" axis and guide the future development of effective therapies by the central-peripheral contrast study. Further research should focus on the causal relationship between the peripheral and central in AD models and patients, which may help gain a better understanding of the pathogenesis of $\mathrm{AD}$ and the potential mechanism of HLJDD in the treatment of AD. 


\section{Supplementary Information}

The online version contains supplementary material available at https://doi. org/10.1186/s13195-021-00779-7.

Additional file 1. Materials and Methods.

Additional file 2: Table S1. Levels and ratios of Bile acids measured in the Tg mice $(n=5)$. Table S2. Peak areas of three main compounds in HLJDD. Table S3. The selected detecting ions, Declustering potential (DP), and collision energy (CE) of neurotransmitters. Table S4. The regression equations and linear range of NTs. Table S5. The selected detecting ions, declustering potential (DP), and collision energy (CE) of UFAs. Table S6. The regression equations and linear range of UFAs. Table S7. The selected detecting ions, declustering potential (DP), and collision energy (CE) of BAs. Table S8. The regression equations and linear range of BAs. Fig. S1. HLJDD altered the overall gut microbiota structure in Tg mice $(n=11)$. (a) Rarefaction analyses; (b) Chao1 index (data expressed as mean \pm SEM); (c) Shannon index (data expressed as mean \pm SEM); (d) ANOSIM analysis, R-value $>0$ means the intra group difference was less than the inter group difference. (e) PLS-DA analyses; ( $f$ ) Bacterial taxonomic profiling in the phylum level of gut microbiota. \#P< 0.05 (comparing with the Mod group). Fig. S2. LEfSe rank plots of differentially abundant microbial clades in gut microbiome $(n=11)$. LDA scores (log 10) for differentially abundant microbial clades in stool among six groups and the threshold on the logarithmic LDA score for discriminative feature is $>$ 3.0. Fig. S3. Venn diagram of the brain (a) and serum (b) showed the shared and unique correlated lipid compounds, in which each ellipse represented the potential lipid markers based on comparisons of the Tg group vs. drug treatment groups. Fig. S4. Profile of HPLCUV chromatograms of mixed standard (a), HLJDD-1st (b) and HLJDD-7th (c) . peaks: 1. Geniposide; 2. Beiberine; 3. Baicalin. The chemical pattern of HLJDD aqueous did not change significantly after 7 days of preparation.

\section{Abbreviations}

5-HT: Serotonin; 5-LOX: 5-Lipoxygenase; AA: Arachidonic acid; AD: Alzheimer's disease; ApoE: Apolipoprotein E; APP: Amyloid precursor protein; $A \beta$ : Amyloid $\beta$-protein; BA: Bile acid; BBB: Blood-brain barrier; BSH: Bile salt hydrolase-rich; Ber: Berberine; CA: Cholic acid;

CDCA: Chenodesoxycholic acid; Cer: Ceramide; CNS: Central nervous system; COX-2: Cyclooxygenase 2; Cp: Cortex phellodendri; DCA: Deoxycholic acid; DHA: Docosahexaenoic acid; Don: Donepezil; ELISA: Enzyme-linked immunosorbent assay; EPA: Eicosapentaenoic acid; FDA: Food and Drug Administration; Fg: Fructus Gardeniae; GABA: $\gamma$-Aminobutyric acid; GLA: $\gamma$ Linolenic acid; GlcCers: Glucosylceramides; Glu: Glutamate; GLUT1: Glucose transporter protein-1; GPX-1: Glutathione peroxidase 1;

HETES: Hydroxyeicosatetraenoic acids; HLJDD: Huanglian Jiedu decoction; $\mathrm{H}-$ L: HLJDD with low dosage; H-H: HLJDD with high dosage; Rs: Radix scutellariae; Rc: Rhizoma coptidis; IBD: Inflammatory bowel disease; IFN$\gamma$ : Interferon- $\gamma ;$ IL-1 $\beta$ : Interleukin-1 $\beta$; IS: Internal standard; Kyn: Kynurenine; LA: Linoleic acid; LCA: Lithocholic acid; LDA: Linear discriminant analysis; LTs: Leukotrienes; MCl: Mild cognitive impairment; MCP-1: Monocyte chemotactic protein 1; MDA: Malonic acid; MIP-2: Murine microphage inflammatory protein-2; MWM: Morris water maze; NO: Nitric oxide; OPLSDA: Orthogonal partial least squares discriminant analysis; PC: Phosphatidylcholine; PE: Phosphatidyl ethanolamine; PEMT: Phosphatidylethanolamine N-methyltransferase; PG: Prostaglandin; Phe: Phenylalanine; PLA2: Phospholipase A2; PLs: Phospholipids; PLSDA: Partial least squares discriminant analysis; ROS: Reactive oxygen species; SCFAs: Short-chain fatty acids; SMs: Sphingomyelins; SOD: Superoxide dismutase; $T_{2}$ DM: Type 2 diabetes mellitus; TCA: Taurocholate; TCDCA: Taurochenodeoxycholate acid; TCM: Traditional Chinese medicine; TDCA: Taurodeoxycholic acid; Tg mice: APP/PS1 transgenic mice; THDCA: Taurohyodeoxycholic acid; TNF-a: Tumour necrosis factor a; Trp: Tryptophan; TUDCA: Tauroursodeoxycholic acid; T-a-MCA: Tauro-amuricholic acid; UDCA: Ursodeoxycholic acid; PUFAs: Polyunsaturated fatty acids; WT: Wild mice

\section{Acknowledgements}

Thanks to Beijing Animals-Science Biotechnology Co., Ltd. for helping raise animals, Thanks for the technical guidance of Basic Medical Experimental Teaching Center of Capital Medical University. Thanks to the Institute of
Chinese Materia Medica, China Academy of Chinese Medical Sciences for providing infrastructure.

\section{Authors' contributions}

Conceived and designed the experiments: Haiyu Zhao, Baolin B. Wrote the paper: Xinru Gu, Haiyu Zhao. Performed the experiments: Xinru Gu, Junyi Zhou, Yanyan Zhou, Xiaorui Fan and Wenya Gao. Analysed the data: Xinru Gu, Hongjie Wang, Nan Si, Wei Ren and Wei Zhao. Contributed to reagents/ materials/analysis tools: Jian Yang, Hongjie Wang. All authors read and approved the final manuscripts.

\section{Funding}

This work was financially supported by the National Natural Science Fund Project under grants [NO.81974523] and [NO. 81573967], and National Science and Technology Major Projects "Major New Drugs Innovation and Development" (No. 2019ZX09201005-006-002 and No. 2019ZX09201005-002005).

\section{Availability of data and materials}

The datasets used and/or analysed during the current study are available from the corresponding author on reasonable request.

\section{Ethics approval and consent to participate}

All experiments and animal care in this study were conducted in accordance with the National Institute of Health guide for the care and use of laboratory animals (NIH Publications No. 8023, revised 1978), and the Provision and General Recommendation of Chinese Experimental Animals Administration Legislation.

\section{Consent for publication}

Not applicable.

\section{Competing interests}

The authors declare that they have no competing interests.

\section{Author details}

${ }^{1}$ Institute of Chinese Materia Medica, China Academy of Chinese Medical Sciences, Beijing 100700, China. ${ }^{2}$ Drug Research Center of Integrated Traditional Chinese and Western Medicine, Affiliated Traditional Chinese Medicine Hospital, Southwest Medical University, Luzhou 646000, China. ${ }^{3}$ Center for Drug Evaluation, National Medical Products Administration, Beijing 100022, China.

Received: 11 May 2020 Accepted: 25 January 2021

Published online: 12 February 2021

\section{References}

1. Alzheimer's Association. 2016 Alzheimer's disease facts and figures. Alzheimers Dement. 2016;12:459-509.

2. Wang $X$, Sun G, Feng T, Zhang J, Huang X, Wang T, et al. Sodium oligomannate therapeutically remodels gut microbiota and suppresses gut bacterial amino acids-shaped neuroinflammation to inhibit Alzheimer's disease progression. Cell Res. 2019;29:787-803.

3. Kondo Y, Kondo F, Asanuma M, Tanaka K, Ogawa N. Protective effect of oren-gedoku-to against induction of neuronal death by transient cerebral ischemia in the C57BL/6 mouse. Neurochem Res. 2000;25:205-9.

4. Xu J, Murakami Y, Matsumoto K, Tohda M, Watanabe H, Zhang S, et al. Protective effect of Oren-gedoku-to (Huang-Lian-Jie-Du-Tang) against impairment of learning and memory induced by transient cerebral ischemia in mice. J Ethnopharmacol. 2000;73:405-13.

5. Fang $H$, Wang $Y$, Yang $T$, Ga $Y$, Zhang $Y$, Liu R, et al. Bioinformatics analysis for the antirheumatic effects of huang-lian-jie-du-tang from a network perspective. Evid Based Complement Alternat Med. 2013;2013:245357.

6. Okamoto H, Chino A, Hirasaki Y, Ueda K, lyo M, Namiki T. Orengedoku-to augmentation in cases showing partial response to yokukan-san treatment: a case report and literature review of the evidence for use of these Kampo herbal formulae. Neuropsychiatr Dis Treat. 2013;9:151-5.

7. Yang Y, Wang HJ, Yang J, Brantner AH, Lower-Nedza AD, Si N, et al. Chemical profiling and quantification of Chinese medicinal formula HuangLian-Jie-Du decoction, a systematic quality control strategy using ultra high performance liquid chromatography combined with hybrid quadrupole- 
orbitrap and triple quadrupole mass spectrometers. J Chromatogr A. 2013; 1321:88-99.

8. Ren W, Zuo R, Wang YN, Wang HJ, Yang J, Xin SK, et al. Pharmacokineticpharmacodynamic analysis on inflammation rat model after oral administration of Huang Lian Jie Du decoction. PLoS One. 2016;11:e0156256.

9. Sun LM, Zhu BJ, Cao HT, Zhang XY, Zhang QC, Xin GZ, et al. Explore the effects of Huang-Lian-Jie-Du-Tang on Alzheimer's disease by UPLC-QTOF/MS-based plasma metabolomics study. J Pharm Biomed Anal. 2018;151:75-83.

10. Durairajan SSK, lyaswamy A, Shetty SG, Kammella AK, Malampati S, et al. A modified formulation of Huanglian-Jie-Du-Tang reduces memory impairments and beta-amyloid plaques in a triple transgenic mouse model of Alzheimer's disease. Sci Rep. 2017;7:6238-51.

11. Tejera D, Mercan D, Sanchez-Caro JM, Hanan M, Greenberg D, Soreq H, et al. Systemic inflammation impairs microglial Abeta clearance through NLRP3 inflammasome. EMBO J. 2019;38:e101064.

12. Calsolaro V, Edison P. Neuroinflammation in Alzheimer's disease: current evidence and future directions. Alzheimers Dement. 2016;12:719-32.

13. Da Mesquita S, Louveau A, Vaccari A, Smirnov I, Cornelison RC, Kingsmore $\mathrm{KM}$, et al. Publisher correction: functional aspects of meningeal lymphatics in ageing and Alzheimer's disease. Nature. 2018;560:185-91.

14. Subhramanyam CS, Wang C, Hu Q, Dheen ST. Microglia-mediated neuroinflammation in neurodegenerative diseases. Semin Cell Dev Biol. 2019;94:112-20.

15. Butterfield DA, Halliwell B. Oxidative stress, dysfunctional glucose metabolism and Alzheimer disease. Nat Rev Neurosci. 2019;20:148-60.

16. Saresella M, Marventano I, Calabrese E, Piancone F, Rainone V, Gatti Andrea, et al. A complex proinflammatory role for peripheral monocytes in Alzheimer's disease. J Alzhermers Dis 2014; 38: 403-413.

17. Cabinio M, Saresella M, Piancone F, LaRosa F, Marventano I, Guerini FS, et al. Association between hippocampal shape, neuroinflammation, and cognitive decline in Alzheimer's disease. J Alzhermers Dis. 2018;66:1131-44.

18. Thériault P, EIAli A, Rivest $S$. The dynamics of monocytes and microglia in Alzheimer's disease. Alzheimers Res Ther. 2015;7:41-51.

19. Hickman SE, Allison EK, Khoury JE. Microglial dysfunction and defective beta-amyloid clearance pathways in aging Alzheimer's disease mice. J Neurosci. 2008:28:8354-60.

20. Minter MR, Taylor JM, Crack PJ. The contribution of neuroinflammation to amyloid toxicity in Alzheimer's disease. J Neurochem. 2016;136:457-74.

21. Lee SH, Suk K. Identification of glia phenotype modulators based on select glial function regulatory signaling pathways. Expert Opin Drug Discov. 2018; 13:627-41.

22. Connolly NMC, Theurey P, Pizzo P. Glucose dysregulation in pre-clinical Alzheimer's disease. Aging. 2019;11:5296-7.

23. Martins RN, Villemagne V, Sohrabi HR, Chatterjee P, Shah TM, Verdile G, et al. Alzheimer's disease: a journey from amyloid peptides and oxidative stress, to biomarker technologies and disease prevention strategies-gains from AIBL and DIAN cohort studies. J Alzheimers Dis. 2018;62:965-92.

24. Di Domenico F, Barone E, Perluigi M, Butterfield DA, Domenico D. The triangle of death in Alzheimer's disease brain: the aberrant cross-talk among energy metabolism, mammalian target of rapamycin signaling, and protein homeostasis revealed by redox proteomics. Antioxid Redox Signal. 2017;26: 364-87.

25. Patrick RP. Role of phosphatidylcholine-DHA in preventing APOE4associated Alzheimer's disease. FASEB J. 2019;33:1554-64.

26. Bazan NG. Lipid signaling in neural plasticity, brain repair, and neuroprotection. Mol Neurobiol. 2005;32:89-103.

27. Whiley L, Sen A, Heaton J, Proitsi P, García-Gómez D, Leung R, et al. Evidence of altered phosphatidylcholine metabolism in Alzheimer's disease. Neurobiol Aging. 2014;35:271-8.

28. Varma VR, Oommen AM, Varma S, Casanova R, An Y, Andrews RM, et al. Brain and blood metabolite signatures of pathology and progression in Alzheimer disease: a targeted metabolomics study. Plos Med. 2018;15:e1002482.

29. Bravo JA, Julio-Pieper M, Forsythe P, Kunze W, Dinan TG, Bienenstock J, et al. Communication between gastrointestinal bacteria and the nervous system. Curr Opin Pharmacol. 2012;12:667-72.

30. Borovikova LV, Ivanova S, Zhang M, Yang H, Botchkina GI, Watkins LR, et al. Vagus nerve stimulation attenuates the systemic inflammatory response to endotoxin. Nature. 2000;405:458-62.

31. Rogers GB, Keating DJ, Young RL, Wong ML, Licinio J, Wesselingh S. From gut dysbiosis to altered brain function and mental illness: mechanisms and pathways. Mol Psychiatry. 2016;21:738-48.
32. Mayer EA, Tillisch $\mathrm{K}$. The brain-gut axis in abdominal pain syndromes. Annu Rev Med. 2011;62:381-96.

33. Grenham S, Clarke G, Cryan JF, Dinan TG. Brain-gut-microbe communication in health and disease. Front Physiol. 2011;2:94-109.

34. Cattaneo A, Cattane N, Galluzzi S, Provasi S, Lopizzo N, Festari C, et al. Association of brain amyloidosis with pro-inflammatory gut bacterial taxa and peripheral inflammation markers in cognitively impaired elderly. Neurobiol of Aging. 2017:49:60-8.

35. Quigley EMM. Microbiota-brain-gut axis and neurodegenerative diseases. Curr Neurol Neurosci Rep. 2017;17:94-103.

36. Macfabe DF, Cain NE, Boon F, Ossenkopp KP, Cain DP. Effects of the enteric bacterial metabolic product propionic acid on object-directed behavior, social behavior, cognition, and neuroinflammation in adolescent rats: relevance to autism spectrum disorder. Behav Brain Res. 2011;217:47-54.

37. Macfabe DF. Short-chain fatty acid fermentation products of the gut microbiome: implications in autism spectrum disorders. Microb Ecol Health Dis. 2012;23:19260-84.

38. Erny $D$, Hrabě de Angelis AL, Jaitin D, Wieghofer P, Staszewski O, David E, et al. Host microbiota constantly control maturation and function of microglia in the CNS. Nat Neurosci. 2015;18:965-77.

39. Tsavkelova EA, Botvinko IV, Kudrin VS, Oleskin AV. Detection of neurotransmitter amines in microorganisms with the use of highperformance liquid chromatography. Dokl Biochem. 2000;372:115-7.

40. Barrett E, Ross RP, O'Toole PW, Fitzgerald GF, Stanton C. Y-Aminobutyric acid production by culturable bacteria from the human intestine. J Appl Microbiol. 2012;113:411-7.

41. Thomas CM, Hong T, van Pijkeren JP, Hemarajata P, Trinh DV, Hu W, et al. Histamine derived from probiotic Lactobacillus reuteri suppresses TNF via modulation of PKA and ERK signaling. PLoS One. 2012;7:e31951.

42. Richard DM, Dawes MA, Mathias CW, Acheson A, Hill-Kapturczak N, Dougherty DM. L-Tryptophan: basic metabolic functions, behavioral research and therapeutic indications. Int J Tryptophan Res. 2009;2:45-60.

43. Erhardt S, Schwieler L, Imbeault S, Engberg G, Erhardt S. The kynurenine pathway in schizophrenia and bipolar disorder. Neuropharmacology. 2017; 112:297-306.

44. Wahlström A, Sayin SI, Marschall HU, Bäckhed F. Intestinal crosstalk between bile acids and microbiota and its impact on host metabolism. Cell Metab. 2016;24:41-50.

45. Melin T, Qi C, Nilsson A. Bile but not chyle lipoprotein is an important source of arachidonic acid for the rat small intestine. Prostag Leukotr Ess. 1996;55:337-43.

46. Nho K, Kueider-Paisley A, MahmoudianDehkordi S, Arnold M, Risacher SL, Louie G. Altered bile acid profile in mild cognitive impairment and Alzheimer's disease: relationship to neuroimaging and CSF biomarkers. Alzheimers Dement. 2019;15:232-44.

47. MahmoudianDehkordi S, Arnold M, Nho K, Ahmad S, Jia W, Xie G, et al. Altered bile acid profile associates with cognitive impairment in Alzheimer's disease-an emerging role for gut microbiome. Alzheimers Dement. 2019;15: 76-92.

48. Cryan JF, Dinan TG. Mind-altering microorganisms: the impact of the gut microbiota on brain and behaviour. Nat Rev Neurosci. 2012;13:701-12.

49. Dinan TG, Cryan JF. Gut instincts: microbiota as a key regulator of brain development, ageing and neurodegeneration. J Physiol. 2017;595:489-503.

50. Dinan TG, Cryan JF. Gut-brain axis in 2016: brain-gut-microbiota axis-mood, metabolism and behaviour. Nat Rev Gastroenterol Hepatol. 2017;14:69-70.

51. Archer RH, Chong R, Maddox IS. Hydrolysis of bile acid conjugates by Clostridium bifermentans. Appl Microbiol Biot. 1982;14:41-5.

52. Sánchez B. Bile acid-microbiota crosstalk in gastrointestinal inflammation and carcinogenesis: a role for bifidobacteria and lactobacilli? Nat Rev Gastroenterology Hepatol. 2018;15:205.

53. Jia W, Xie G, Jia W. Bile acid-microbiota crosstalk in gastrointestinal inflammation and carcinogenesis. Nat Rev Gastroenterol Hepatol. 2018;15: $111-28$.

54. Ridlon JM, Dae-Joong K, Hylemon PB. Bile salt biotransformations by human intestinal bacteria. J Lipid Res. 2006;47:241-59.

55. Jones BV, Begley M, Hill C, Gahan CG, Marchesi JR. Functional and comparative metagenomic analysis of bile salt hydrolase activity in the human gut microbiome. Proc Natl Acad Sci U S A. 2008;105:13580-5.

56. Gilliland SE, Speck ML. Deconjugation of bile acids by intestinal lactobacilli. Appl Environ Microbiol. 1977;33:15-8. 
57. Brandscheid C, Schuck F, Reinhardt S, Schäfer KH, Pietrzik CU, Grimm M, et al. Altered gut microbiome composition and tryptic activity of the 5xFAD Alzheimer's mouse model. J Alzheimers Dis. 2016;56:775-88.

58. Park JY, Choi J, Lee Y, Lee JE, Lee EH, Kwon HJ, et al. Metagenome analysis of bodily microbiota in a mouse model of Alzheimer disease using bacteriaderived membrane vesicles in blood. Exp Neurobiol. 2017;26:369-79.

59. Lee HJ, Lee KE, Kim JK, Kim DH. Suppression of gut dysbiosis by Bifidobacterium longum alleviates cognitive decline in 5XFAD transgenic and aged mice. Sci Rep. 2019;9:11814-26.

60. Cheignon C, Tomas M, Bonnefont-Rousselot D, Faller P, Hureau C, Collin F, et al. Oxidative stress and the amyloid beta peptide in Alzheimer's disease Redox Biol. 2018;14:450-64.

61. Zhang CE, Wei W, Liu YH, Peng JH, Tian Q, Liu GP, et al. Hyperhomocysteinemia increases beta-amyloid by enhancing expression of gamma-secretase and phosphorylation of amyloid precursor protein in rat brain. Am J Pathol. 2009;174:1481-91.

62. Tapia-Rojas C, Lindsay CB, Montecinos-Oliva C, Arrazola MS, Retamales RM, Bunout $D$, et al. Is L-methionine a trigger factor for Alzheimer's-like neurodegeneration?: changes in Abeta oligomers, tau phosphorylation, synaptic proteins, Wnt signaling and behavioral impairment in wild-type mice. Mol Neurodegener. 2015;10:62-79.

63. King E, O'Brien JT, Donaghy P, Morris C, Barnett N, Olsen K, et al. Peripheral inflammation in prodromal Alzheimer's and Lewy body dementias. J Neurol Neurosurg Psychiatry. 2018;89:339-45.

64. Zheng C, Zhou XW, Wang JZ. The dual roles of cytokines in Alzheimer's disease: update on interleukins, TNF- $\alpha$, TGF- $\beta$ and IFN- $\gamma$. Transl Neurodegener. 2016:5:1-15.

65. Baruch K, Deczkowska A, David E, Castellano JM, Miller O, Kertser A, et al. Aging Aging-induced type I interferon response at the choroid plexus negatively affects brain function. Science. 2014;346:89-93.

66. Baruch K, Ron-Harel N, Gal H, Deczkowska A, Shifrut E, Ndifon W, et al. CNSspecific immunity at the choroid plexus shifts toward destructive Th2 inflammation in brain aging. Proc Natl Acad Sci U S A. 2013;110:2264-9.

67. Kunis $\mathrm{G}$, Baruch K, Miller O, Schwartz M. Immunization with a myelinderived antigen activates the Brain's choroid plexus for recruitment of immunoregulatory cells to the CNS and attenuates disease progression in a mouse model of ALS. J Neurosci. 2015;35:6381-93.

68. Lee WJ, Liao YC, Wang YF, Lin IF, Wang SJ, Fuh JL. Plasma MCP-1 and cognitive decline in patients with Alzheimer's disease and mild cognitive impairment: a two-year follow-up study. Sci Rep. 2018;8:1280-8.

69. Janelsins MC, Mastrangelo MA, Oddo S, LaFerla FM, Federoff HJ, Bowers WJ. Early correlation of microglial activation with enhanced tumor necrosis factor-alpha and monocyte chemoattractant protein-1 expression specifically within the entorhinal cortex of triple transgenic Alzheimer's disease mice. J Neuroinflammation. 2005;2:23-35.

70. El Gaamouch F, Jing P, Xia J, Cai D. Alzheimer's disease risk genes and lipid regulators. J Alzheimers Dis. 2016;23:15-29.

71. Haughey NJ, Bandaru W, Bae M, Mattson MP. Roles for dysfunctional sphingolipid metabolism in Alzheimer's disease neuropathogenesis. Biochim Biophys Acta. 1801;2010:878-86.

72. de Wilde MC, Vellas B, Girault E, Yavuz AC, Sijben JW. Lower brain and blood nutrient status in Alzheimer's disease: results from meta-analyses. Alzheimers Dement. 2017;3:416-31.

73. Orešič M, Hyötyläinen T, Herkka SK, Sysi-Aho M, Mattila I, Seppänan-Laakso T, et al. Metabolome in progression to Alzheimer's disease. Transl Psychiatry. 2011;1:e57.

74. Li D, Misialek JR, Boerwinkle E, Gottesman RF, Sharrett AR, Mosley TH, et al, Prospective associations of plasma phospholipids and mild cognitive impairment/dementia among African Americans in the ARIC neurocognitive study. Alzheimers Dement (Amst). 2017;6:1-10.

75. Tönnies E, Trushina ED. Oxidative stress, synaptic dysfunction, and Alzheimer's disease. J Alzheimers Dis. 2017:57:1105-21.

76. van Wijk N, Broersen LM, de Wilde MC, Hageman RJ, Groenendijk M, Sijben JW et al. Targeting synaptic dysfunction in Alzheimer's disease by administering a specific nutrient combination. J Alzheimers Dis. 2014;38:459-79.

77. Gonzalez-Dominguez R, Garcia-Barrera T, Gomez-Ariza JL. Combination of metabolomic and phospholipid-profiling approaches for the study of Alzheimer's disease. J Proteome. 2014;104:37-47.

78. Cunnane SC, Schneider JA, Tangney C, Tremblay-Mercier J, Fortier M, Bennett DA, et al. Plasma and brain fatty acid profiles in mild cognitive impairment and Alzheimer's disease. J Alzheimers Dis. 2012;29:691-7.
79. Cunnane SC, Plourde M, Pifferi F, Bégin M, Féart C, Barberger-Gateau P. Fish, docosahexaenoic acid and Alzheimer's disease. Prog Lipid Res. 2009;48:239-56.

80. Fraser T, Tayler H, Love S. Fatty acid composition of frontal, temporal and parietal neocortex in the normal human brain and in Alzheimer's disease. Neurochem Res. 2010;35:503-13.

81. Amtul Z, Uhrig M, Wang L, Rozmahel RF, Beyreuther K. Detrimental effects of arachidonic acid and its metabolites in cellular and mouse models of Alzheimer's disease: structural insight. Neurobiol Aging. 2012;33:e821-31.

82. Manev H. 5-Lipoxygenase gene polymorphism and onset of Alzheimer's disease. Med Hypotheses. 2000;54:75-6.

83. Snowden SG, Ebshiana AA, Hye A, An Y, Pletnikova O, O'Brien R, et al. Association between fatty acid metabolism in the brain and Alzheimer disease neuropathology and cognitive performance: a nontargeted metabolomic study. Plos Med. 2017;14:e1002266.

84. Youdim KA, Martin A, Joseph JA. Essential fatty acids and the brain: possible health implications. Int J Dev Neurosci. 2000;18:383-99.

85. La Rosa F, Clerici M, Ratto D, Occhinegro A, Licito A, Romeo M, et al. The gut-brain axis in Alzheimer's disease and omega-3. A critical overview of clinical trials. Nutrients. 2018;10(9):1267-23.

86. Kimura I, Inoue D, Maeda T, Hara T, Ichimura A, Miyauchi S, et al. Shortchain fatty acids and ketones directly regulate sympathetic nervous system via G protein-coupled receptor 41 (GPR41). Proc Natl Acad Sci U S A. 2011; 108:8030-5.

87. Nøhr MK, Pedersen MH, Gille A, Egerod KL, Engelstoft MS, Husted AS, et al. GPR41/FFAR3 and GPR43/FFAR2 as cosensors for short-chain fatty acids in enteroendocrine cells vs FFAR3 in enteric neurons and FFAR2 in enteric leukocytes. Endocrinology. 2013;154:3552-64.

88. Giannelli V, Di Gregorio V, lebba V, Giusto M, Schippa S, Merli M, et al. Microbiota and the gut-liver axis: bacterial translocation, inflammation and infection in cirrhosis. World J Gastroenterol. 2014;20:16795-810.

89. Collins SM, Michael S, Premysl B. The interplay between the intestinal microbiota and the brain. Nat Rev Microbiol. 2012;10:735-42.

90. Scott KA, Ida M, Peterson VL, Prenderville JA, Moloney GM, Izumo T, et al. Revisiting Metchnikoff: age-related alterations in microbiota-gut-brain axis in the mouse. Brain Behav Immun. 2017;65:20-32.

91. Jiang $H$, Ling $Z$, Zhang $Y$, Mao $H, M a ~ Z$, Yin $Y$, et al. Altered fecal microbiota composition in patients with major depressive disorder. Brain Behav Immun. 2015:48:186-94.

92. Bonfili L, Cecarini V, Berardi S, Scarpona S, Suchodolski JS, Nasuti C, et al. Microbiota modulation counteracts Alzheimer's disease progression influencing neuronal proteolysis and gut hormones plasma levels. Sci Rep. 2017;7:2426-47.

93. Harach T, Marungruang N, Duthilleul N, Cheatham V, Mc Coy KD, Frisoni G, et al. Reduction of Abeta amyloid pathology in APPPS1 transgenic mice in the absence of gut microbiota. Sci Rep. 2017;7:41802-15.

94. Dinan TG, Cryan JF. Gut instincts: microbiota as a key regulator of brain development, ageing and neurodegeneration. J Physiol. 2016;595:489-503.

95. Staley C, Weingarden AR, Khoruts A, Sadowsky MJ. Interaction of gut microbiota with bile acid metabolism and its influence on disease states. Appl Microbiol Biot. 2017;101:47-64.

96. Jia W, Rajani C, Kaddurah-Daouk R, Li H. Expert insights: The potential role of the gut microbiome-bile acid-brain axis in the development and progression of Alzheimer's disease and hepatic encephalopathy. Med Res Rev. 2020;40:1496-1507.

97. Quinn M, McMillin M, Galindo C, Frampton G, Pae HY, DeMorrow S. Bile acids permeabilize the blood brain barrier after bile duct ligation in rats via Rac1-dependent mechanisms. Dig Liver Dis. 2014:46:527-34.

98. Monte MJ, Marin JJG, Antelo A, Vazquez-Tato J. Bile acids: chemistry, physiology, and pathophysiology. World J Gastroenterol. 2009;15:804-16.

99. Swann JR, Want EJ, Geier FM, Spagou K, Wilson ID, Sidaway JE, et al. Systemic gut microbial modulation of bile acid metabolism in host tissue compartments. Proc Natl Acad Sci U S A. 2011;108:4523-30.

100. Pan X, Elliott CT, McGuinness B, Passmore P, Kehoe PG, Hölscher C, et al. Metabolomic profiling of bile acids in clinical and experimental samples of Alzheimer's disease. Metabolites. 2017;7:28-40.

101. François A, Rioux Bilan A, Quellard N, Fernandez B, Janet T, Chassaing D, et al. Longitudinal follow-up of autophagy and inflammation in brain of APPswePS1dE9 transgenic mice. J Neuroinflamm. 2014;11:139-53.

102. Guest J, Garg M, Bilgin A, Grant R. Relationship between central and peripheral fatty acids in humans. Lipids Health Dis. 2013:12:79-87. 
103. Shen L, Liu L, Ji HF. Alzheimer's disease histological and behavioral manifestations in transgenic mice correlate with specific gut microbiome state. J Alzheimers Dis. 2016;56:385-90.

104. Ghaisas S, Maher J, Kanthasamy A. Gut microbiome in health and disease: linking the microbiome-gut-brain axis and environmental factors in the pathogenesis of systemic and neurodegenerative diseases. Pharmacol Ther. 2016;158:52-62.

105. Chen M, Liao Z, Lu B, Wang M, Lin L, Zhang S, et al. Huang-Lian-Jie-Dudecoction ameliorates hyperglycemia and insulin resistant in association with gut microbiota modulation. Front Microbiol. 2018;9:2380-96.

106. Vogt NM, Kerby RL, Dill-McFarland KA, Harding SJ, Merluzzi AP, Johnson SC, et al. Gut microbiome alterations in Alzheimer's disease. Sci Rep. 2017;7: 563-74.

107. Sun NX, Tong $L T$, Liang $T$, Wang $L L$, Liu LY, Zhou XR, et al. Effect of oat and Tartary buckwheat-based food on cholesterol-lowering and gut microbiota in hypercholesterolemic hamsters. J Oleo Sci. 2019;68:251-9.

108. Jia W, Xie G, Jia W. Bile acid-microbiota crosstalk in gastrointestinal inflammation and carcinogenesis. Nat Rev Gastro Hepat. 2017;15:111-28.

\section{Publisher's Note}

Springer Nature remains neutral with regard to jurisdictional claims in published maps and institutional affiliations.

Ready to submit your research? Choose BMC and benefit from:

- fast, convenient online submission

- thorough peer review by experienced researchers in your field

- rapid publication on acceptance

- support for research data, including large and complex data types

- gold Open Access which fosters wider collaboration and increased citations

- maximum visibility for your research: over $100 \mathrm{M}$ website views per year

At BMC, research is always in progress.

Learn more biomedcentral.com/submissions 